\title{
A DOZEN NEW GALAXIES CAUGHT IN THE ACT: GAS STRIPPING AND EXTENDED EMISSION LINE REGIONS IN THE COMA CLUSTER*
}

\author{
Masafumi Yagi $^{1}$, Michitoshi Yoshida ${ }^{2}$, Yutaka Komiyama ${ }^{1}$, Nobunari Kashikawa ${ }^{1}$, Hisanori Furusawa ${ }^{3}$, \\ Sadanori Okamura ${ }^{4,5}$, Alister W. Graham ${ }^{6}$, Neal A. Miller $^{7}$, David Carter $^{8}$, Bahram Mobasher ${ }^{9}$, and Shardha Jogee ${ }^{10}$

\section{ABSTRACT}

We present images of extended $\mathrm{H} \alpha$ clouds associated with 14 member galaxies in the Coma cluster obtained from deep narrowband imaging observations with the Suprime-Cam at the Subaru Telescope. The parent galaxies of the extended $\mathrm{H} \alpha$ clouds are distributed farther than $0.2 \mathrm{Mpc}$ from the peak of the X-ray emission of the cluster. Most of the galaxies are bluer than $g-r \approx 0.5$ and they account for $57 \%$ of the blue $(g-r<0.5)$ bright $(r<17.8 \mathrm{mag})$ galaxies in the central region of the Coma cluster. They reside near the red- and blueshifted edges of the radial velocity distribution of Coma cluster member galaxies. Our findings suggest that most of the parent galaxies were recently captured by the Coma cluster potential and are now infalling toward the cluster center with their disk gas being stripped off and producing the observed $\mathrm{H} \alpha$ clouds.

Key words: galaxies: clusters: individual (Abell 1656) - galaxies: evolution - galaxies: structure

Online-only material: color figures

\section{INTRODUCTION}

Clusters of galaxies are ideal laboratories for investigating environmental effects on galaxy evolution. It is well known that galaxies residing in the central region of clusters are generally deficient in gas (e.g., Giovanelli \& Haynes 1985; Gavazzi 1987; Solanes et al. 2001; Boselli \& Gavazzi 2006; Bravo-Alfaro et al. 2009). This indicates that some gas removal mechanisms work quite efficiently in the cluster center. Recently, van der Wel et al. (2010) studied the shape and magnitude of quiescent galaxies in Sloan Digital Sky Survey (SDSS) and found that galaxies in dense environments include gas-removed spiral galaxies, adding to the evidence for rapid gas removal. A number of possible physical processes have been proposed as explanations of efficient gas removal in clusters: ram pressure (Gunn \& Gott 1972) or viscous stripping (Nulsen 1982), tidal stripping by galaxy-galaxy interactions (Toomre \& Toomre 1972), or the cluster potential (Byrd \& Valtonen 1990), evaporation (Cowie \& Songaila 1977), and to a lesser degree, galaxy harassment (Moore et al. 1996).

One critical clue for understanding the gas removal is detection of the stripped gas. Recent deep $\mathrm{H} \alpha$ imaging observations revealed that some of the galaxies in clusters are accompanied by $\mathrm{H} \alpha$ clouds. In the nearby cluster Abell 1367, Gavazzi et al. (2001) found extended ionized gas regions associated with starburst irregular galaxies. The sizes of the regions are $75 \times 8$ and $50 \times 8 \mathrm{kpc}$. Yoshida et al. (2002) discovered another extended emission line region $(\sim 35 \mathrm{kpc})$ in the Virgo cluster, which was found to be a part of $110 \times 25 \mathrm{kpc}$ of H I gas (Oosterloo \& van

\footnotetext{
* Based on data collected at the Subaru Telescope, which is operated by the National Astronomical Observatory of Japan.
}

Gorkom 2005). Yagi et al. (2007) reported a narrow and long $(60 \times 2 \mathrm{kpc}) \mathrm{H} \alpha$ emitting region associated with a galaxy of the Coma cluster. Sun et al. (2007) found a $\sim 40 \mathrm{kpc} \mathrm{H} \alpha$ tail of a galaxy in Abell 3627. The galaxy also had a $\sim 70 \mathrm{kpc} \mathrm{X-ray} \mathrm{tail.}$ Sun et al. (2010) investigated the galaxy to show two $\sim 80 \mathrm{kpc}$ tails, and also reported one more galaxy with a $>20 \mathrm{kpc} \mathrm{H} \alpha$ tail in the cluster. Kenney et al. (2008) detected $120 \mathrm{kpc} \mathrm{H} \alpha$ filaments from NGC 4438, a spiral galaxy in the Virgo cluster. Yoshida et al. (2008) reported a galaxy with detached $\mathrm{H} \alpha$ clouds with young blue stars in the Coma cluster, which resembles two galaxies in $z \sim 0.2$ clusters reported by Cortese et al. (2007). These $\mathrm{H} \alpha$ cloud findings imply that gas removal mechanisms to form such extended emission line regions are common in clusters of galaxies. However, it is not yet clear how common this phenomenon is.

The Coma cluster is the nearest rich cluster of galaxies and is the best target for investigating the $\mathrm{H} \alpha$ clouds in the cluster environment with a deep and spatially resolved observation. We have performed a deep $\mathrm{H} \alpha$ narrowband imaging survey of the Coma Cluster using the Suprime-Cam at the Subaru Telescope and have already reported some of the prominent clouds detected (Yagi et al. 2007; Yoshida et al. 2008). Here, we report significant, extended $\mathrm{H} \alpha$ clouds around an additional 12 galaxies, increasing the sample to a total of 14 . This is the first paper in a series providing a statistical discussion of extended emission line regions in the central region of the Coma cluster. The nature of the clouds and physical interpretations will be presented in subsequent papers.

We assume that the distance modulus of the Coma cluster is $(m-M)_{0}=35.05$ (Kavelaars et al. 2000) and $\left(h_{0}, \Omega_{M}\right.$, $\left.\Omega_{\lambda}\right)=(0.71,0.27,0.73)$ (Larson et al. 2010). Under these 
Table 1

Summary of Mosaicked Images and Observation Log

\begin{tabular}{|c|c|c|c|c|}
\hline Filter & PSF size & $\mathrm{SB}_{\lim }\left(\mathrm{ABmag} \operatorname{arcsec}^{-2}\right)$ & Date (UT) & Exposure \\
\hline \multirow[t]{4}{*}{$B$} & $1^{\prime \prime} 06$ & 28.6 & 2006 Apr 28 & $2 \times 450 \mathrm{~s}$ \\
\hline & & & 2006 May 3 & $3 \times 450 \mathrm{~s}$ \\
\hline & & & 2007 May 13 & $4 \times 600 \mathrm{~s}$ \\
\hline & & & 2009 May 26 & $6 \times 600 \mathrm{~s}$ \\
\hline \multirow[t]{8}{*}{$R$} & $0^{\prime \prime} 75$ & 28.1 & 2006 Apr 28 & $11 \times 300 \mathrm{~s}$ \\
\hline & & & 2006 May 3 & $1 \times 60 \mathrm{~s}$ \\
\hline & & & 2007 May 11 & $1 \times 60 \mathrm{~s}$ \\
\hline & & & 2007 May 13 & $12 \times 300 \mathrm{~s}$ \\
\hline & & & 2007 May 14 & $5 \times 360 \mathrm{~s}$ \\
\hline & & & 2007 May 15 & $1 \times 300 \mathrm{~s}$ \\
\hline & & & 2009 May 26 & $4 \times 300 \mathrm{~s}$ \\
\hline & & & 2009 May 27 & $4 \times 300 \mathrm{~s}$ \\
\hline \multirow[t]{3}{*}{$i$} & $1^{\prime \prime} .10$ & 27.5 & 2006 Apr 28 & $4 \times 240 \mathrm{~s}$ \\
\hline & & & 2006 May 3 & $1 \times 240 s+2 \times 120 s$ \\
\hline & & & 2009 May 25 & $6 \times 300 \mathrm{~s}$ \\
\hline \multirow[t]{5}{*}{$N B$} & $0^{\prime \prime} 76$ & 27.5 & 2006 Apr 28 & $8 \times 1800 \mathrm{~s}$ \\
\hline & & & 2006 May 3 & $3 \times 120 \mathrm{~s}$ \\
\hline & & & 2007 May 12 & $9 \times 1800 \mathrm{~s}$ \\
\hline & & & 2007 May 15 & $1 \times 300 \mathrm{~s}$ \\
\hline & & & 2009 May 26 & $4 \times 900 \mathrm{~s}$ \\
\hline
\end{tabular}

Note. $\mathrm{SB}_{\lim }$ represents $1 \sigma$ fluctuation of flux in an aperture of 2 arcsec diameter.

assumptions, the angular diameter distance is $97.47 \mathrm{Mpc}$, and 1 arcsec corresponds to $0.473 \mathrm{kpc}$.

\section{OBSERVATION AND DATA REDUCTION}

We observed a field around the Coma cluster center $(\alpha, \delta)(\mathbf{J} 2000)=(12: 59: 26,+27: 44: 16)$ with the Suprime-Cam (Miyazaki et al. 2002) at the Subaru Telescope in 2006-2009. The field position and orientation are shown in Figure 1, and the observations are summarized in Table 1 . The observed region was within $1 \mathrm{Mpc}$ from the center, which is less than half of the virial radius of the Coma cluster (2.8 Mpc; Okabe et al. 2010). We used three broadband filters $(B, R, i)$, and a narrowband filter $(N-A-L 671$, hereafter $N B)$. The $N B$-filter was designed for observing $\mathrm{H} \alpha$ emitting objects in the Coma cluster at $z=0.0225$, and has bell-shaped transmission with a central wavelength of $6712 \AA$ and an FWHM of $120 \AA$ (Yagi et al. 2007; Yoshida et al. 2008).

The imaging data were reduced in a standard manner. Overscan subtraction, flat-fielding, distortion correction, background subtraction, and mosaicking were performed using another implementation (nekosoft; Yagi et al. 2002) of the standard Suprime-Cam reduction software. Satellite trails and contaminated pixels with saturated photons were masked manually before mosaicking. The flux calibration was performed using the seventh Data Release (Abazajian et al. 2009, hereafter DR7) of the SDSS photometric catalog. The details are described in the Appendix.

The limiting surface brightness and point-spread function (PSF) sizes of the final $B, R, i$, and $N B$ images are listed in Table 1. The limiting surface brightness was estimated by photometry of randomly sampled apertures 2 arcsec in diameter. We used the median of an absolute deviation (MAD) multiplied by a factor $f=1.482602$ as a robust estimator of the rms. The factor was calculated by solving erf $(1 / f)=3 / 4$

$$
\int_{-\infty}^{1 / f} \frac{1}{\sqrt{2 \pi}} e^{-\frac{x^{2}}{2}} d x=3 / 4
$$

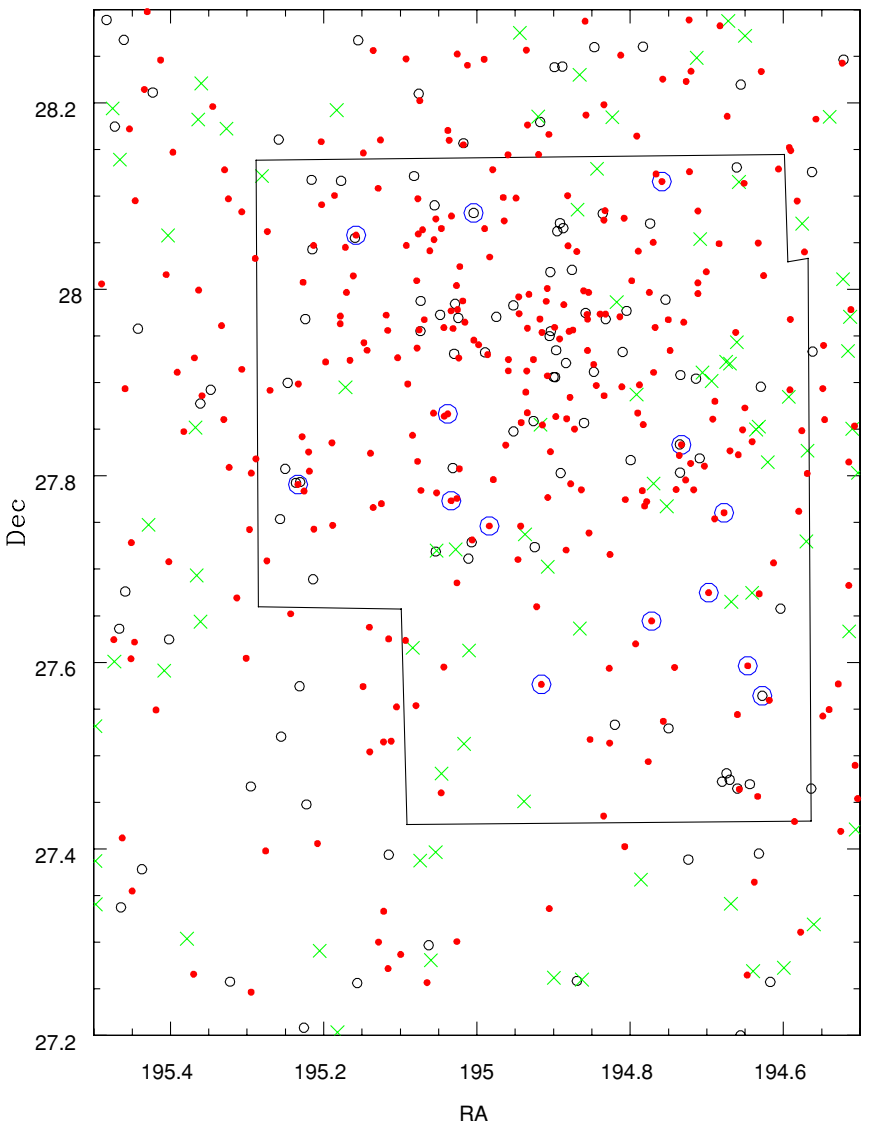

Figure 1. Coma cluster area. The solid line represents the region observed in the present study. The symbols are SDSS galaxies of $r<18$. The red filled circles have a spectroscopic redshift between 0.015 and 0.035 , the green crosses have a redshift out of the range, and the black open circles have no SDSS spectroscopic information. The blue large open circles show the parent galaxies of the ionized clouds. Two of the parents did not have SDSS redshift and were confirmed to be Coma members by spectroscopy of Hectospec.

(A color version of this figure is available in the online journal.) 

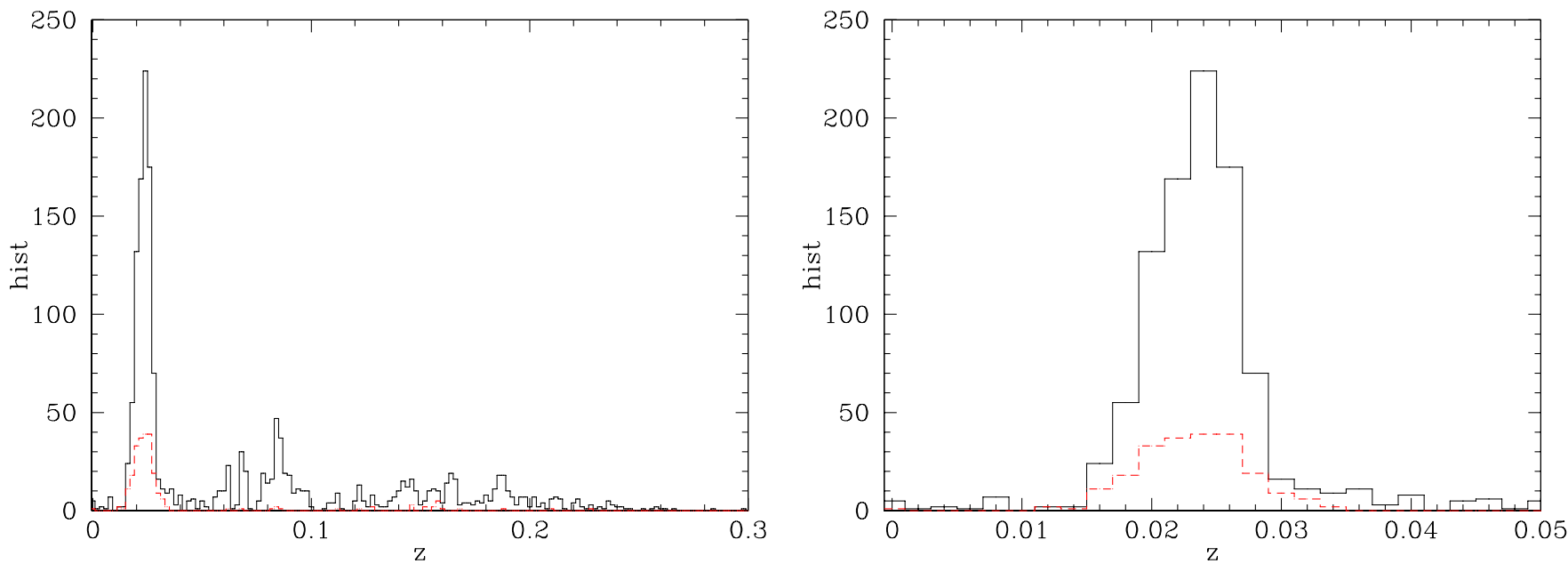

Figure 2. Redshift distribution of SDSS galaxies brighter than $r=17.8 \mathrm{mag}$ that were located in our field (red broken line histogram) and within a $2^{\circ}$ radius from the cluster center (black solid line histogram). The right figure is a zoom around $z=0.025$.

(A color version of this figure is available in the online journal.)
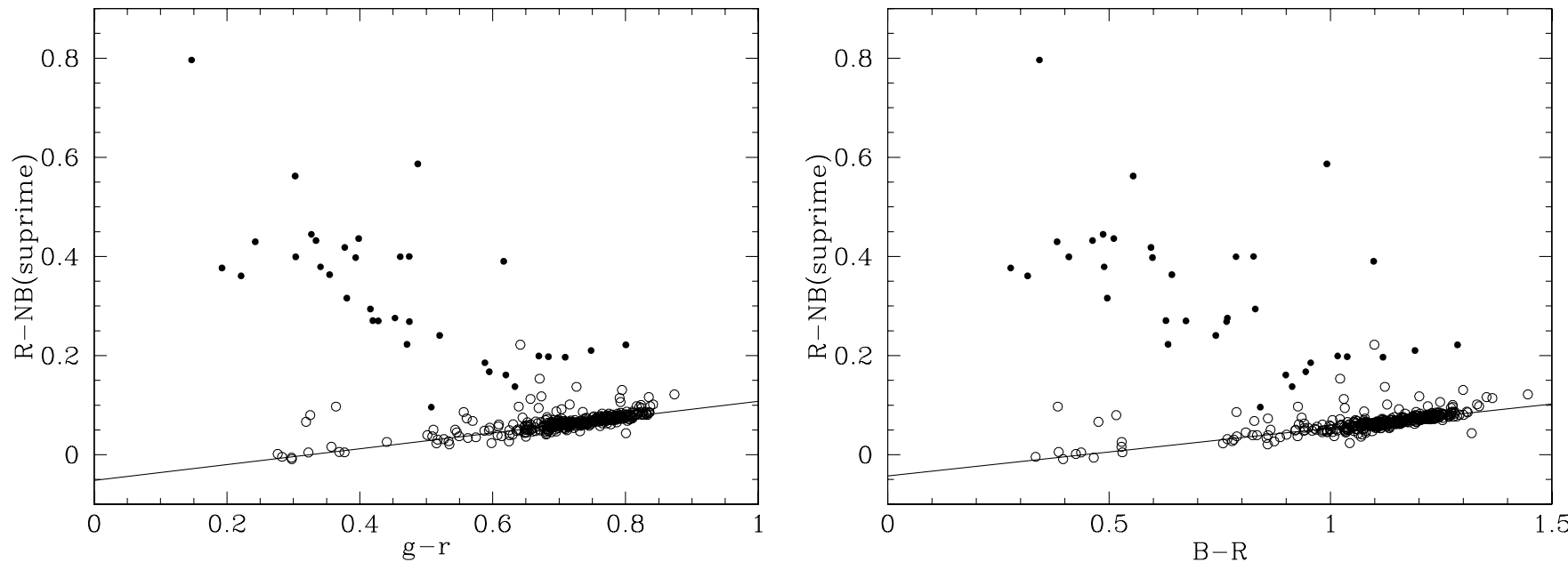

Figure 3. $R-N B$ color of cluster member galaxies estimated from the SDSS spectrum as a function of SDSS $g-r$ color using fiberMag (left) and Suprime-Cam $B-R$ color estimated from the SDSS spectrum of each galaxy (right). The dots represent those with $\mathrm{EW}(\mathrm{H} \alpha)>10 \AA$ and the open circles represent other galaxies. The solid line is the regression line for $\mathrm{EW}(\mathrm{H} \alpha)<1$ A galaxies.

and $f \times$ MAD equals the rms, when the error followed a Gaussian distribution.

\section{DETECTION OF H $\alpha$ CLOUDS}

\subsection{Ho Image}

Figure 2 shows the redshift distribution of galaxies brighter than $r=17.8 \mathrm{mag}$ within a radius of $2^{\circ}$ from the cluster center and those in our observed field. Redshifts were taken from the SDSS DR7 spectroscopic catalog. Based on Figure 2, we defined the galaxies with $0.015<z<0.035$ as Coma member galaxies.

To detect $\mathrm{H} \alpha$ clouds, we subtracted the $R$-band image from the $N B$ image. Here, we refer to the $R$-band subtracted $N B$-band image as the $\mathrm{H} \alpha$ image. First, the typical $R-N B$ color of Coma members was estimated by multiplying the filter response to the SDSS spectra of galaxies with $0.015<z<0.035$. There are 395 galaxies in the redshift range with SDSS spectra in our observed field. The results are shown in Figure 3, where $R-N B$ color is plotted as a function of SDSS color $(g-r)$ and SuprimeCam color $(B-R)$. As the $R-N B$ is calculated from the SDSS spectrum, we use fiberMag for $(g-r)$, which corresponds to the color of the central 3 arcsec aperture. The large dots represent the galaxies with equivalent width $\mathrm{EW}(\mathrm{H} \alpha)>10 \AA$ in SDSS. Figure 3 shows that the $R-N B$ color of galaxies with $\mathrm{EW}(\mathrm{H} \alpha)<$ $10 \AA$ correlates with the $g-r$ and $B-R$ color. The lines are the regression line for $\mathrm{EW}(\mathrm{H} \alpha)<1 \AA$ galaxies:

$$
R-N B=0.15(g-r)_{\mathrm{SDSS}}-0.043
$$

or

$$
R-N B=0.097(B-R)-0.043 .
$$

This relation is useful in the analysis of emission line galaxies. The main purpose of this study, however, was to detect nebulous features, where neither $g-r$ information nor our $B-R$ color are necessarily available with a reliable signal-to-noise ratio. Therefore, we used the median color of these member galaxies, $R-N B=0.065 \mathrm{mag}$, as the color of a typical Coma passive galaxy, i.e., a galaxy with no $\mathrm{H} \alpha$ emission. We scaled the $R$ band image so that such galaxies had values of 0 in the $\mathrm{H} \alpha$ image. As slight ( $<1$ pixel, 0.2 arcsec) positional shifts between the $R$-band and $N B$-band remained in some regions, sub-pixel alignment was performed by the method of Yagi (2010) before subtraction. 
Table 2

List of Parent Galaxies ${ }^{\mathrm{a}}$

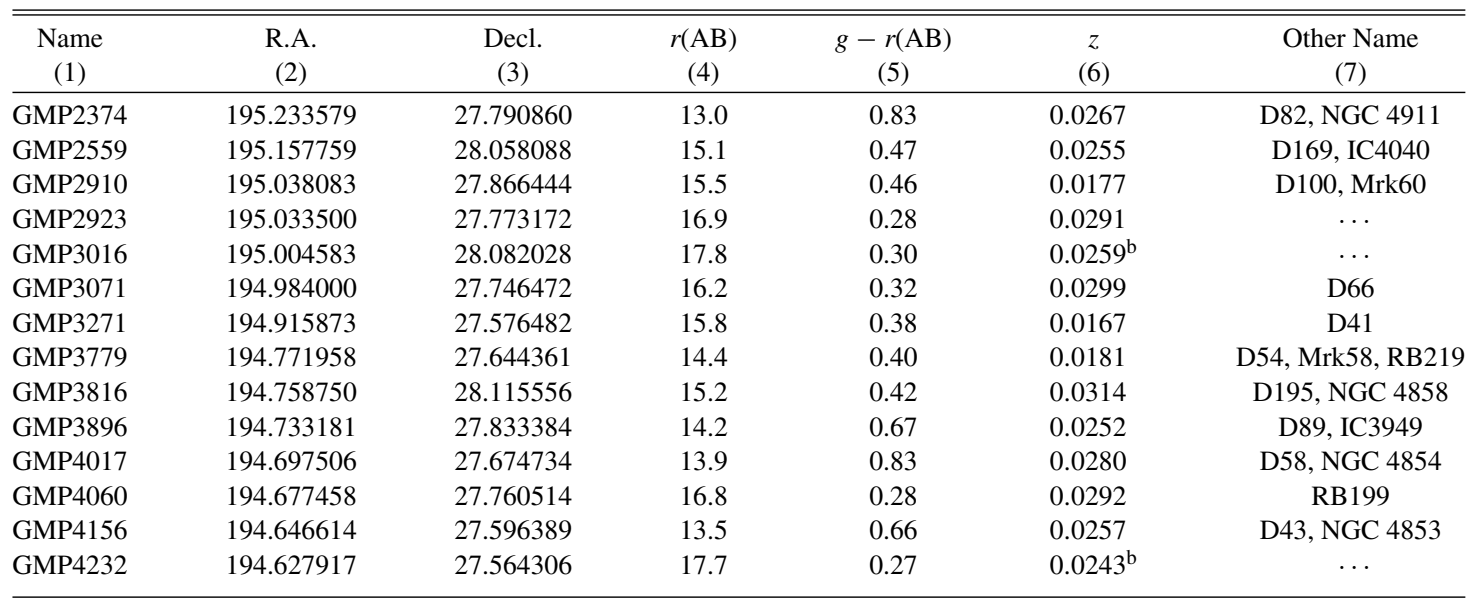

Notes. The data are taken from SDSS and NED

a (1) GMP name, (2) R.A. (J2000), (3) Decl. (J2000), (4) $r$-band model magnitude from SDSS DR7, (5) $g-r$ color from SDSS, (6) redshift from SDSS, and (7) other name.

${ }^{b}$ Hectospec redshift.

We also constructed a three-color image from $B, R$, and $N B$. The colors of the member galaxies in $B, R, i$, and $N B$ in the Suprime-Cam filter system were calculated from SDSS spectra. We adopted a scale such that those galaxies with $B-R=1.0$, $R-N B=0.065$, and $N B-i=0.2$ were gray in the three-color image.

In the $\mathrm{H} \alpha$ image, clouds were searched by visual inspection. Ghosts of bright stars and residuals of background subtractions were carefully checked and excluded. The correlation of $R-N B$ and color (Figure 3) suggested that red galaxies would have residuals in the $\mathrm{H} \alpha$ image, while blue galaxies would have negative values. This point was verified using $B$ - and $i$-band data. If a region was bright in $N B$ and also red in the $B-R$ and $R-i$ colors, it was regarded as a residual. If the color was blue, however, it was regarded as a star-forming region and was included in our sample of $\mathrm{H} \alpha$ clouds.

\subsection{List of Clouds and Parameter Measurements}

All of the extended $\mathrm{H} \alpha$ clouds detected here are shown in Figures 4(a)-(n) and listed in Table 2. We named the clouds after possible associated galaxies (hereafter, parent galaxies), using the galaxy IDs of Godwin et al. (1983). The contrast of three-color composites (left images) was selected arbitrarily according to the brightness of the parent galaxy, while the color balance was fixed. A pure red color represented $\mathrm{H} \alpha$ emission without any stellar light, purple represented $\mathrm{H} \alpha$ emission with some stellar light, and blue color represented light from young blue stars without any $\mathrm{H} \alpha$ emission. In Figures 4(e) and (1), we can see blue filaments from the parent galaxy in the left image, while no $\mathrm{H} \alpha$ emission is present in the right image. These are the "fireball" features reported by Yoshida et al. (2008).

The contrast of the $\mathrm{H} \alpha$ image (right images) is the same for Figures 4(a)-(n). Therefore, the gray colors could be directly compared among the figures to measure the apparent $\mathrm{H} \alpha$ brightness. The green line in the $\mathrm{H} \alpha$ image represents the isophote of the $\mathrm{H} \alpha$-image at $2.5 \times 10^{-18} \mathrm{erg} \mathrm{s}^{-1} \mathrm{~cm}^{-2} \operatorname{arcsec}^{-2}$, assuming that the clouds have the same redshift as the parent. The red contour represents the isophote of our $R$-band image, which was useful for comparing the extension of clouds with stellar light. The $R$-band isophote was chosen so that the region inside it contained the same area as the aperture of the $r$ band half-light radius (PetroR50) of SDSS, because the bright galaxies in our $R$-band image had saturated pixels and we were not able to measure the radius in our data directly. In addition, the isophotal treatment was appropriate as the parents had distorted shapes.

We performed spectroscopic observations of some of the clouds using the Faint Object Camera and Spectrograph (FOCAS; Kashikawa et al. 2002) in multi-object spectroscopy (MOS) mode on 2006 June 23, and 2009 May 19 UT. The case of GMP2910(D100) was reported previously in Yagi et al. (2007). Other cloud results will be described in M. Yoshida et al. (2010, in preparation). In this study, we used the spectroscopic result indicating that four clouds had similar redshifts to their respective parent galaxies (GMP2559, GMP2910, GMP2923, and GMP4060). In Figure 4(d), GMP2945, which lies just to the west of GMP2923, seems to be the parent of the clouds, but GMP2945 had a markedly different redshift $(z=0.0207)$ from the clouds $(z \sim 0.028)$. We identified GMP2923( $z=0.0291)$ as the parent and GMP2945 as a chance overlap. Twelve of fourteen parent galaxies had SDSS spectral information, and the other two had a Hectospec redshift (R. O. Marzke et al. 2010, in preparation). There were six galaxies that had both SDSS and Hectospec redshifts. They were consistent within $\sim 30 \mathrm{~km} \mathrm{~s}^{-1}$.

Table 3 shows the extension of the $\mathrm{H} \alpha$ clouds relative to the parent galaxy and the surface brightness of the clouds measured in the $\mathrm{H} \alpha$ image. The $\mathrm{H} \alpha$ total flux was calculated by assuming that the cloud had the same redshift as the parent galaxy, and its mix of [N $\mathrm{NI}] 6548,6583$ was similar to that of the GMP2910 clouds (Yagi et al. 2007). We adopted a value of $[\mathrm{N}$ II $] 6583 / \mathrm{H} \alpha=0.5$. Other emission lines, such as [O I] 6300 and $\left[\mathrm{S}_{\mathrm{II}}\right] 6716,6731$, were out of the passband in the redshift of $0.015<z<0.035$ and were therefore negligible. As the wavelength dispersion of the emission lines of the GMP2910 cloud in the FOCAS data was $\sim 3.8 \AA$ (Yagi et al. 2007), we adopted a value for the model to compute the $\mathrm{H} \alpha$ flux. As it includes broadening by an instrumental profile, the value would be overestimated for $\mathrm{H} \alpha$ imaging data. However, the effect of the dispersion was found to be negligible. The dispersion of $3.8 \AA$ corresponded to velocity dispersion of $\sim 170 \mathrm{~km} \mathrm{~s}^{-1}$, but 

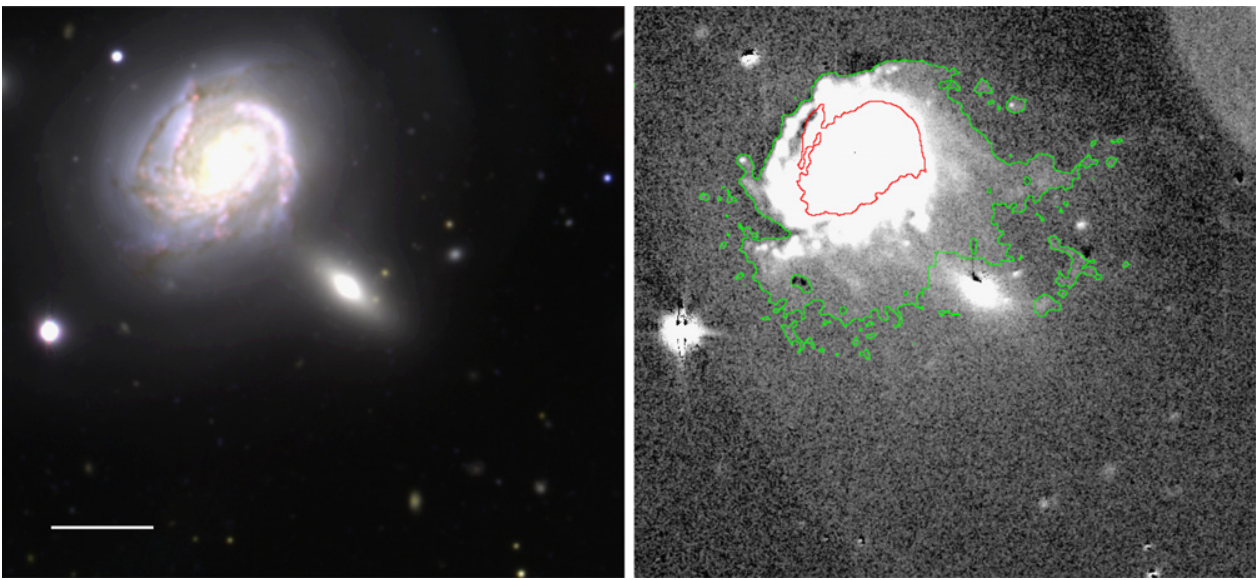

(a)
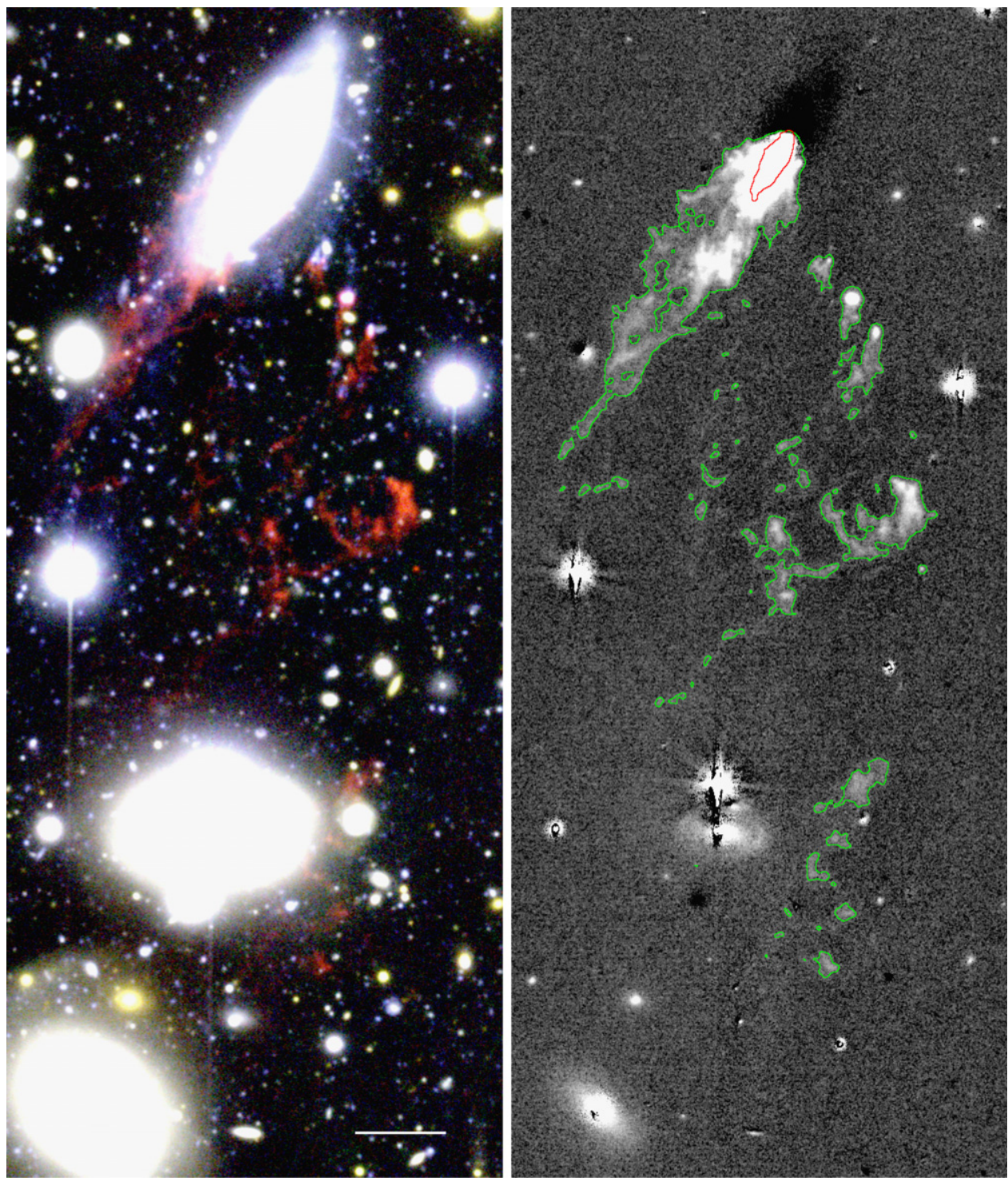

(b)

Figure 4. Left: $B, R$, and $N B$ three-color composite of GMP2374. North is up and east is to the left. The relative $B, R$, and $N B$ scales are set so that typical Coma galaxies without $\mathrm{H} \alpha$ emission have gray color. The contrast is arbitrary. The white bar represents a scale of $10 \mathrm{kpc}(21$ arcsec). The pure red color represents $\mathrm{H} \alpha$ emission without any stellar light, the purple color represents $\mathrm{H} \alpha$ emission with some stellar light, and the blue color represents young blue stars without $\mathrm{H} \alpha$ emission. Right: $N B-R(\mathrm{H} \alpha)$ image of the same region as the left image. The green contour represents the isophote of $2.5 \times 10^{-18} \mathrm{erg} \mathrm{s}^{-1} \mathrm{~cm}^{-2}$ arcsec ${ }^{-2}$. The red contour represents the isophote of the $R$-band image which corresponds to the SDSS $r$-band petroR50 (the radius containing $50 \%$ of the Petrosian flux). A typical Coma galaxy without $\mathrm{H} \alpha$ emission disappears in this image. White indicates $N B$ flux excess and black indicates $N B$ flux deficiency. As discussed in the text, the excess/deficiency correlated with the color of the underlying stellar components. Redder and bluer underlying stellar components made $N B$ excess and $N B$ deficiency, respectively. The contrast of $N B-R$ image (right) is the same for all the parents ((a)-(n)). ((b)-(n)): (b), same as (a), but of GMP2559, (c) GMP2910, (d) GMP2923, (e) GMP3016, (f) GMP3071, (g) GMP3271, (h) GMP3779, (i) GMP3816, (j) GMP3896, (k) GMP4017, (l) GMP4060, (m) GMP4156, and (n) GMP4232. 

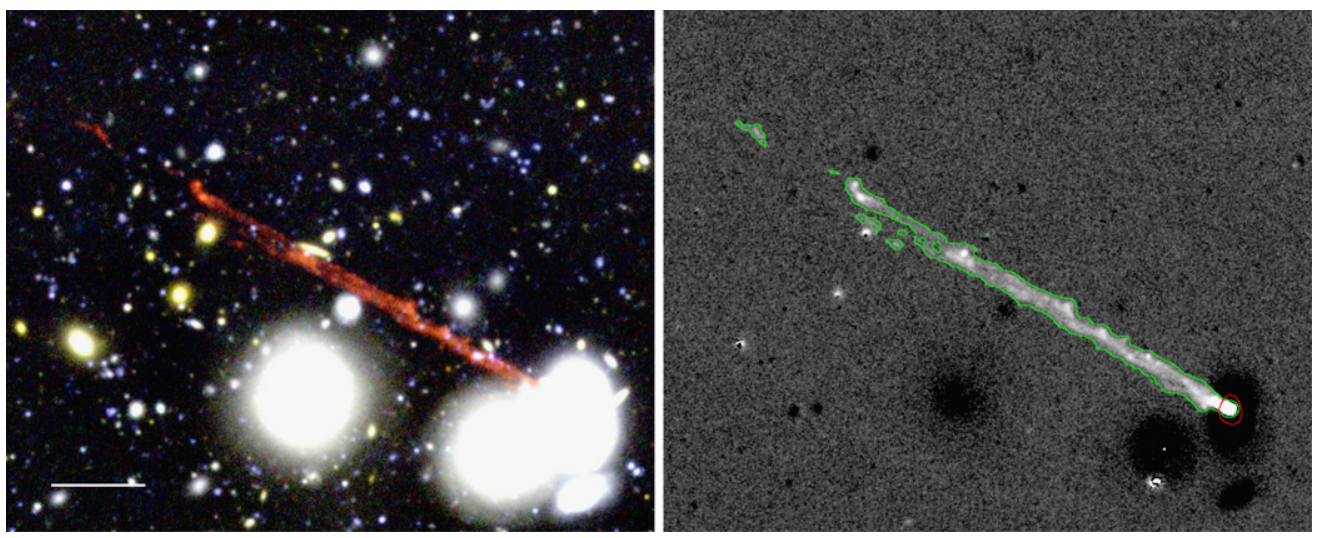

(c)

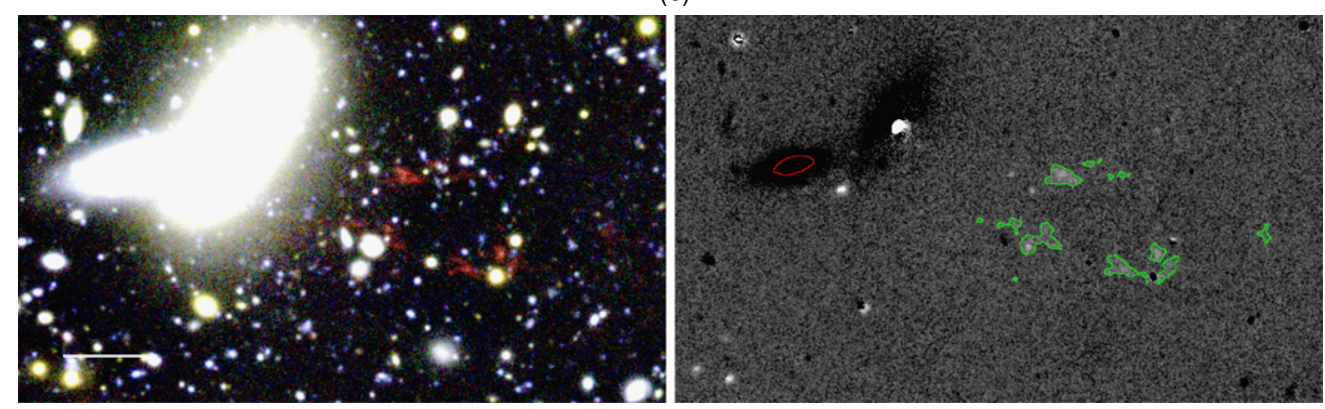

(d)
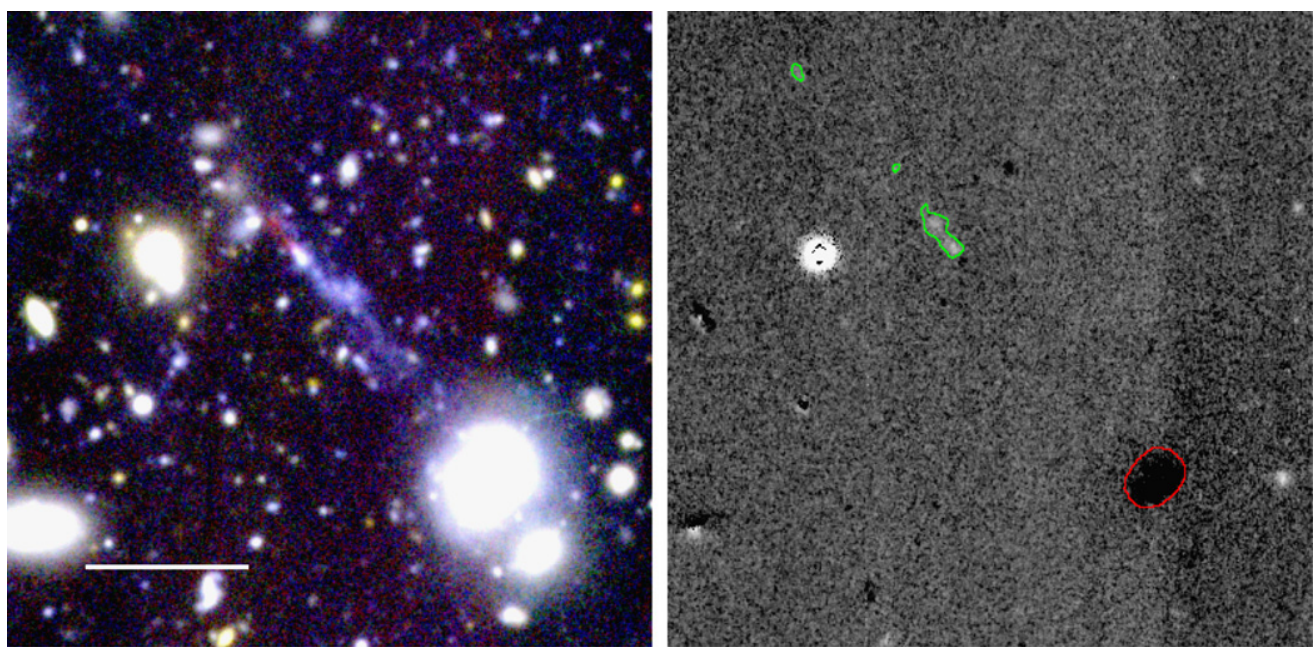

(e)
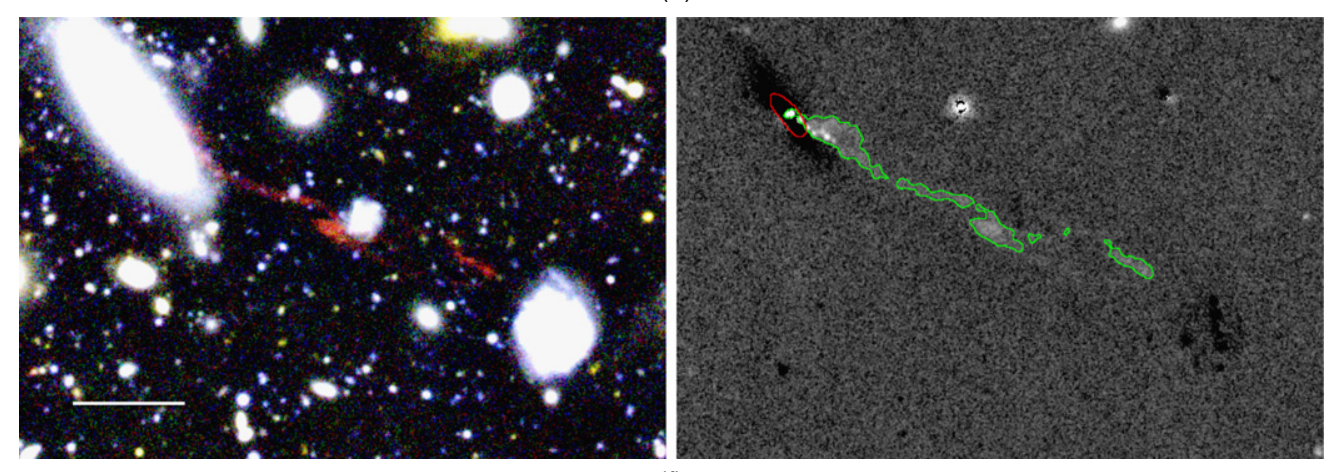

(f)

Figure 4. (Continued) 

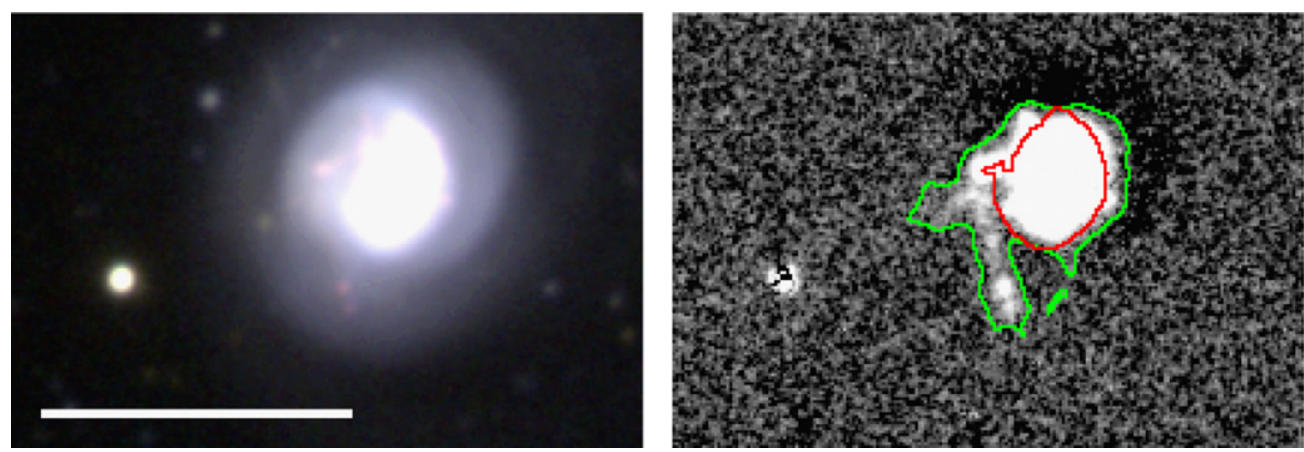

(g)
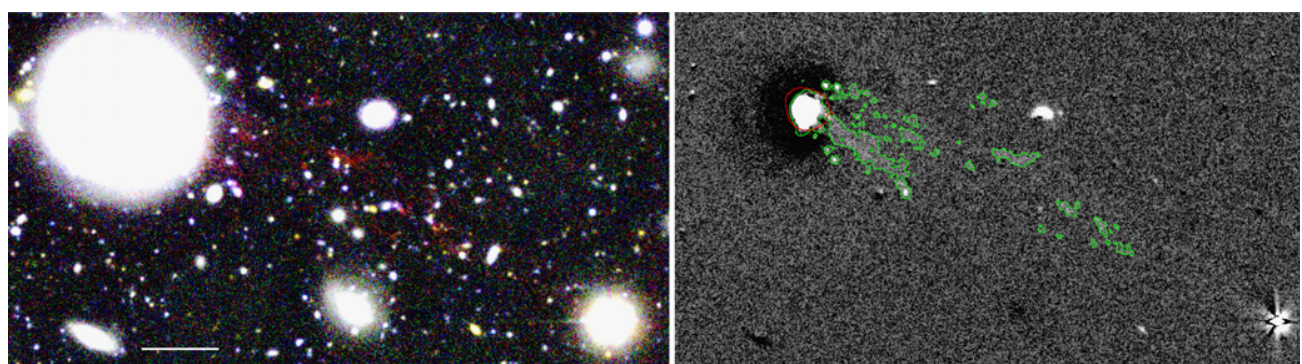

(h)
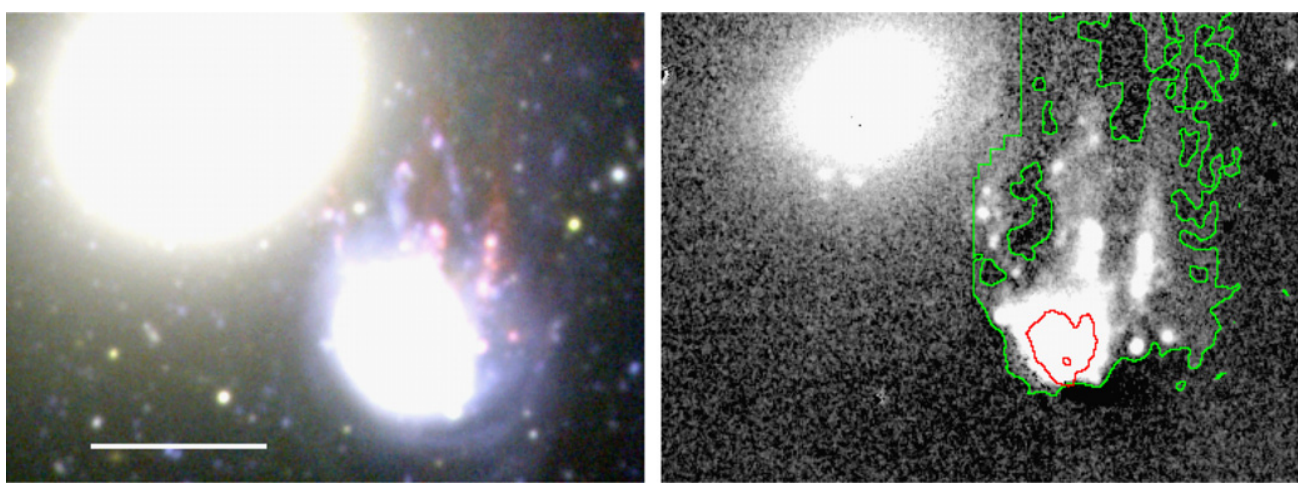

(i)
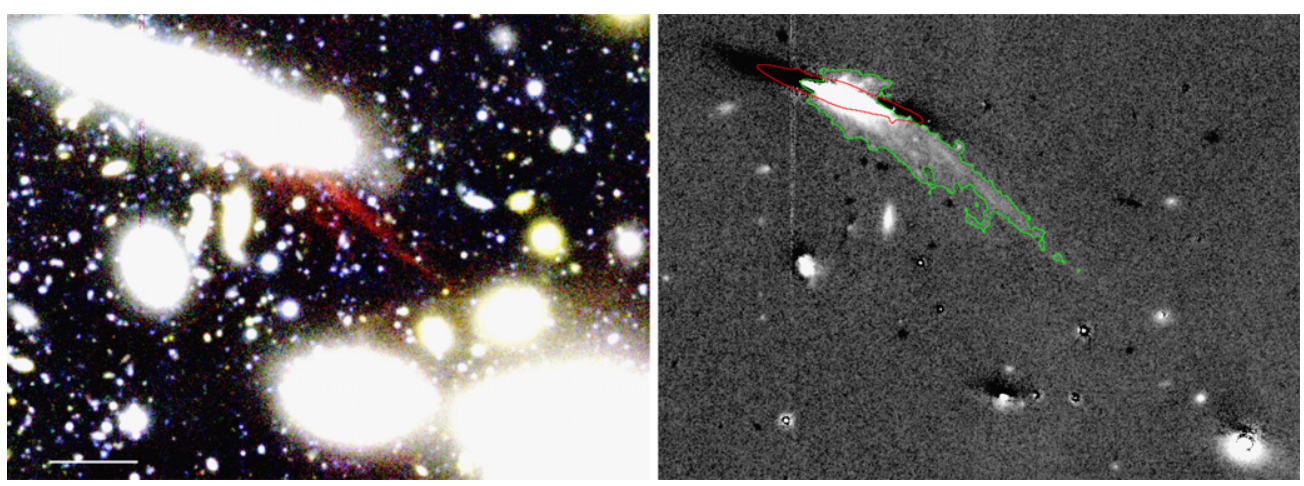

(j)

Figure 4. (Continued)

even if we assume a dispersion of $10 \mathrm{~km} \mathrm{~s}^{-1}$, the change in $\mathrm{H} \alpha$ magnitude is less than $0.01 \mathrm{mag}$. We defined the $\mathrm{H} \alpha$ magnitude $(\operatorname{mag}(\mathrm{H} \alpha))$ as the continuum subtracted $N B$ magnitude.

$\operatorname{mag}(\mathrm{H} \alpha)_{A B}=-2.5 \log \left[\frac{\int R_{N B} f_{\nu} d v-k \int R_{R} f_{\nu} d v}{\int R_{N B} d v}\right]-48.6$, where $R_{N B}$ and $R_{R}$ are the transmission of the filters, $f_{v}$ is the flux density, and $k$ is the correction factor so that

$$
\int R_{N B} f_{v} d v=k \int R_{R} f_{v} d v
$$

for objects with median color without $\mathrm{H} \alpha$ emission, $R-N B=$ 0.065 . We calculated the $\operatorname{mag}(\mathrm{H} \alpha)$ from the flux in the $N B-R$ image with a zero-point $N B$-band magnitude. It should be noted that the $\mathrm{H} \alpha$ magnitude is not an $N B-R$ color but the $\mathrm{AB}$ magni- 


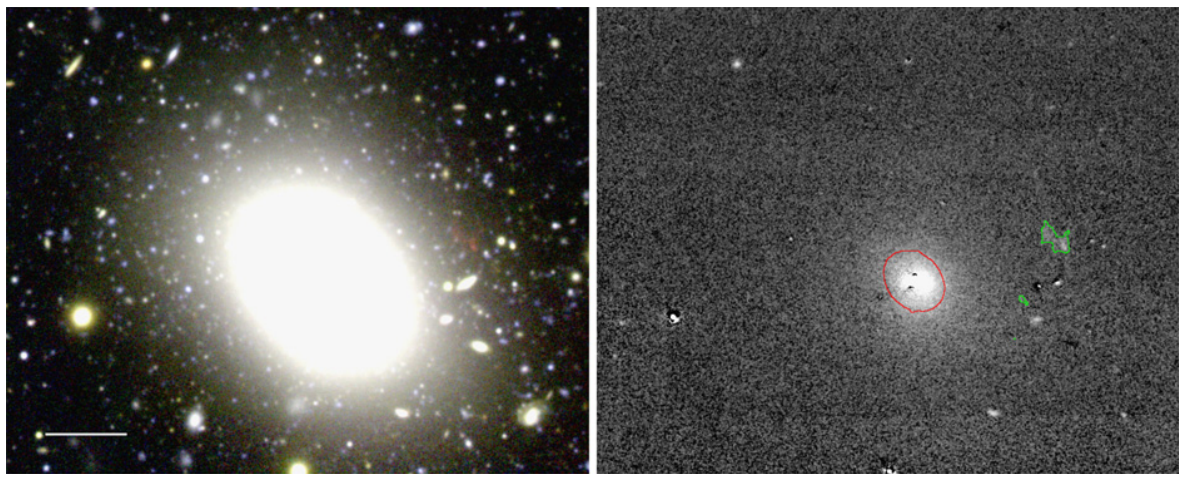

(k)
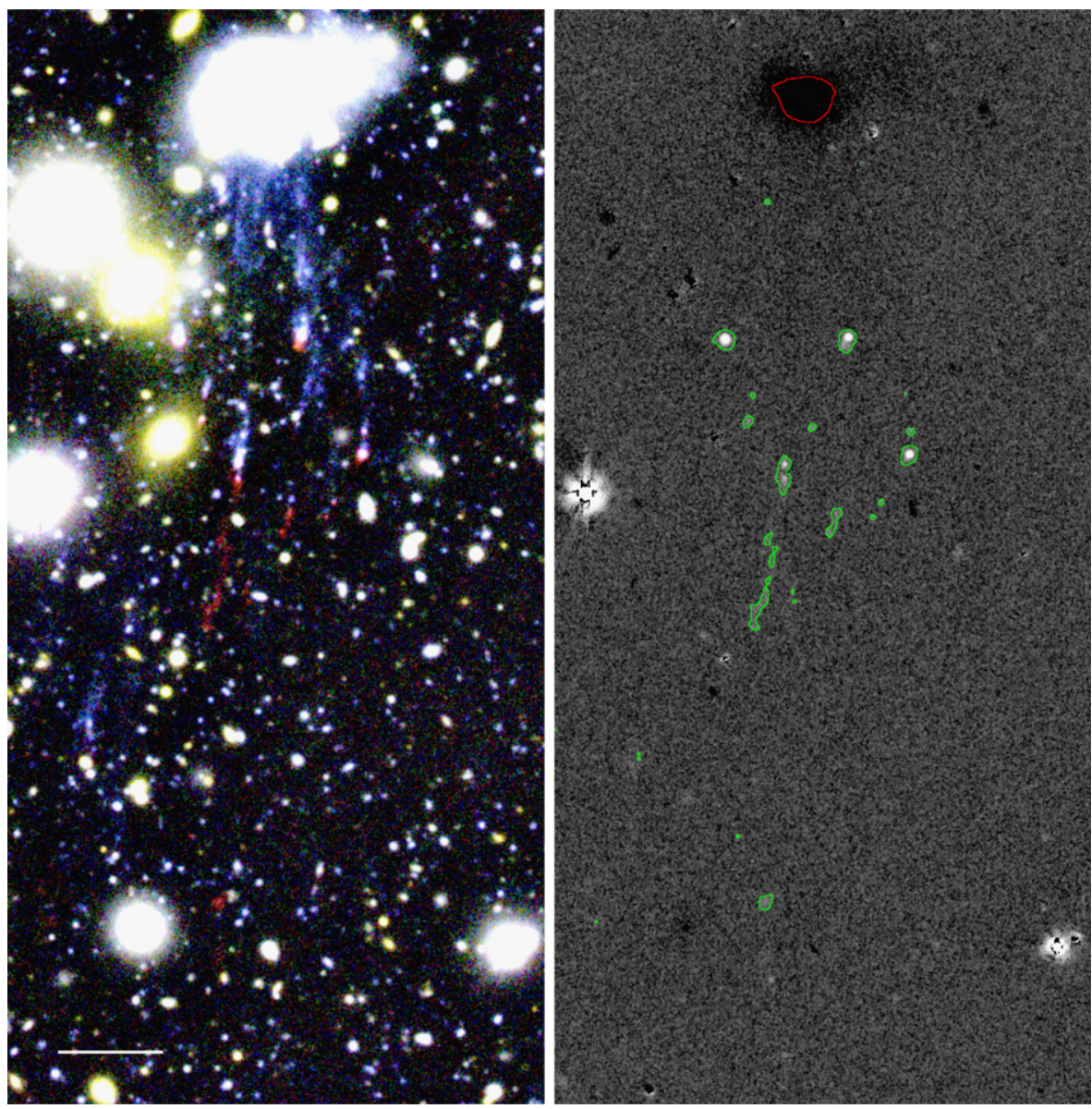

(l)
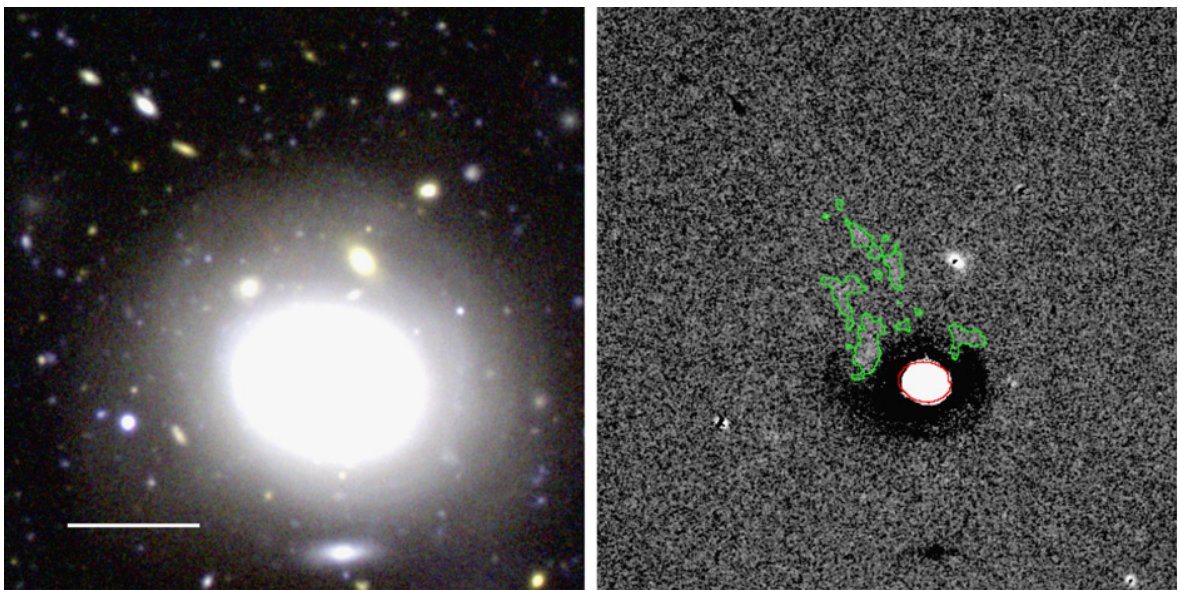

(m)

Figure 4. (Continued) 

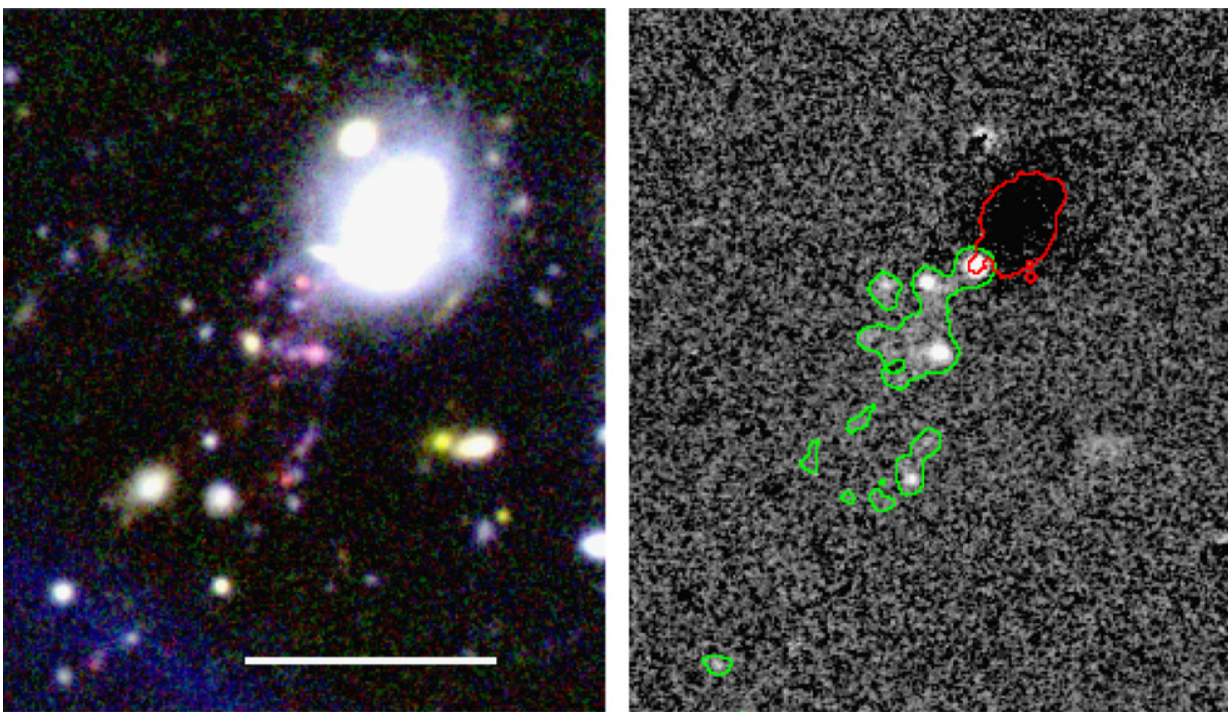

(n)

Figure 4. (Continued)

Table 3

Properties of $\mathrm{H} \alpha$ Clouds $^{\mathrm{a}}$

\begin{tabular}{ccccccccc}
\hline \hline $\begin{array}{c}\text { Name } \\
(1)\end{array}$ & $\begin{array}{c}\text { Area } \\
(2)\end{array}$ & $\begin{array}{c}\text { Extension } \\
(3)\end{array}$ & $\begin{array}{c}\text { Position Angle } \\
(4)\end{array}$ & $\begin{array}{c}\text { SB(R) } \\
(5)\end{array}$ & $\begin{array}{c}\text { Peak SB(H } \alpha) \\
(6)\end{array}$ & $\begin{array}{c}\text { Total mag(H } \alpha) \\
(7)\end{array}$ & $\begin{array}{c}\text { H } \alpha \text { Flux } \\
(8)\end{array}$ & $\begin{array}{c}\text { Type } \\
(9)\end{array}$ \\
\hline GMP2374 & 2350 & 28 & 249 & 21.3 & 18.6 & 15.0 & $2.3 \times 10^{-13}$ & 1 \\
GMP2559 & 1962 & 90 & 183 & 22.9 & 19.7 & 15.7 & $1.1 \times 10^{-13}$ & 1 \\
GMP2910 & 498 & 60 & 60 & 23.0 & 19.2 & 17.7 & $1.9 \times 10^{-14}$ & 2 \\
GMP2923 & 130 & 54 & 254 & 25.9 & 25.8 & 21.6 & $6.6 \times 10^{-16}$ & 3 \\
GMP3016 & 21 & 34 & 40 & 26.5 & 25.7 & 22.9 & $1.4 \times 10^{-16}$ & 3 \\
GMP3071 & 168 & 35 & 247 & 23.5 & 22.9 & 20.8 & $1.5 \times 10^{-15}$ & 2 \\
GMP3271 & 124 & 4.7 & 157 & 21.2 & 20.8 & 18.1 & $1.4 \times 10^{-14}$ & 1 \\
GMP3779 & 436 & 47 & 245 & 21.7 & 19.1 & 16.9 & $3.7 \times 10^{-14}$ & 2 \\
GMP3816 & 584 & 21 & 333 & 22.4 & 19.5 & 16.4 & $1.0 \times 10^{-13}$ & 1 \\
GMP3896 & 585 & 34 & 234 & 21.2 & 20.0 & 16.8 & $3.9 \times 10^{-14}$ & 2 \\
GMP4017 & 38 & 20 & 288 & 25.3 & 25.6 & 22.5 & $2.4 \times 10^{-16}$ & $3($ red) \\
GMP4060 & 116 & 81 & 165 & 26.2 & 23.4 & 20.9 & $1.2 \times 10^{-15}$ & 3 \\
GMP4156 & 118 & 15 & 26 & 22.4 & 25.8 & 21.3 & $6.5 \times 10^{-16}$ & $3($ red $)$ \\
GMP4232 & 92 & 22 & 151 & 25.0 & 22.8 & 20.7 & $1.0 \times 10^{-15}$ & 3 \\
\hline
\end{tabular}

Notes. ${ }^{\text {a }}$ Column 1: name of the parent, Column 2: area above the threshold $\left(\operatorname{arcsec}^{2}\right)$, Column 3: distance from the most distant part of cloud to the center of the parent $(\mathrm{kpc})$, Column 4: position angle of the farthest part relative to the center of the parent, north $=0$ and east $=90(\mathrm{deg})$, Column 5: mean surface brightness of the clouds in $R$-band (mag $\left.\operatorname{arcsec}^{-2}\right)$ including background continuum, Column 6: peak $\mathrm{H} \alpha$ surface brightness ( $m a g \operatorname{arcsec}^{-2}$ ), Column 7: total $\mathrm{H} \alpha$ (isophotal) magnitude (mag), Column 8: estimated total $\mathrm{H} \alpha$ flux ( $\mathrm{erg} \mathrm{s}^{-1} \mathrm{~cm}^{-2}$ ), and Column 9: apparent morphological type of the clouds (see the text).

tude measured in the $\mathrm{H} \alpha$ image. That is, the $N B-R$ color corresponds to $\mathrm{EW}(\mathrm{H} \alpha)$, while $\mathrm{H} \alpha$ magnitude corresponds to $L(\mathrm{H} \alpha)$.

The measurement of flux was performed in the fixed isophote of a smoothed image. The $\mathrm{H} \alpha$ image was smoothed with a Gaussian kernel of $\sigma=2.5$ pixel, so that the noise of each pixel was suppressed. The isophotal threshold for the analysis was set to $2.5 \times 10^{-18} \mathrm{erg} \mathrm{s}^{-1} \mathrm{~cm}^{-2} \operatorname{arcsec}^{-2}$ at the redshift of the parent galaxy. As the transmission of the $N B$ filter has a bell-shaped curve, the transmission at $\mathrm{H} \alpha$ changes according to the redshift. This effect was corrected when the threshold was calculated. The detected objects were examined with three-color images. Possible overlapping objects, such as foreground stars, distant galaxies, or a redder galaxy core were removed manually. Even after this screening, some of the clouds had errors due to the stellar color of the parent galaxy. In addition, some included light from $\mathrm{H}$ II regions in the parent galaxy. The examined detection map was used as a mask, and the total flux and peak surface brightness were measured in the masked $\mathrm{H} \alpha$ image. The same mask was applied to the $R$ band and the mean surface brightness in the $R$ band $\left(\mathrm{SB}_{R}\right)$, which is an indicator of the extent of contamination of the $\mathrm{H} \alpha$ emission by continuum light, was measured. The measured values are summarized in Table 3.

\subsection{Note on GMP4017 Clouds}

The H $\alpha$ clouds of GMP4017 lie along the line of the clouds of GMP4060 and are slightly elongated in the north-south direction (Figure 5). This implies that they may not be associated with GMP4017, but with GMP4060. If the GMP4017 clouds were in fact an extension of the GMP4060 clouds, the length of the GMP4060 cloud would be $144 \mathrm{kpc}$. As the redshift of GMP4017(0.0280) is similar to that of GMP4060(0.0292), it is difficult to identify the physical association from spectroscopy.

H I observations may uncover the origin of the clouds of GMP4017. However, no H I emission has been detected around GMP4017 by previous deep H I surveys (Bravo-Alfaro et al. 


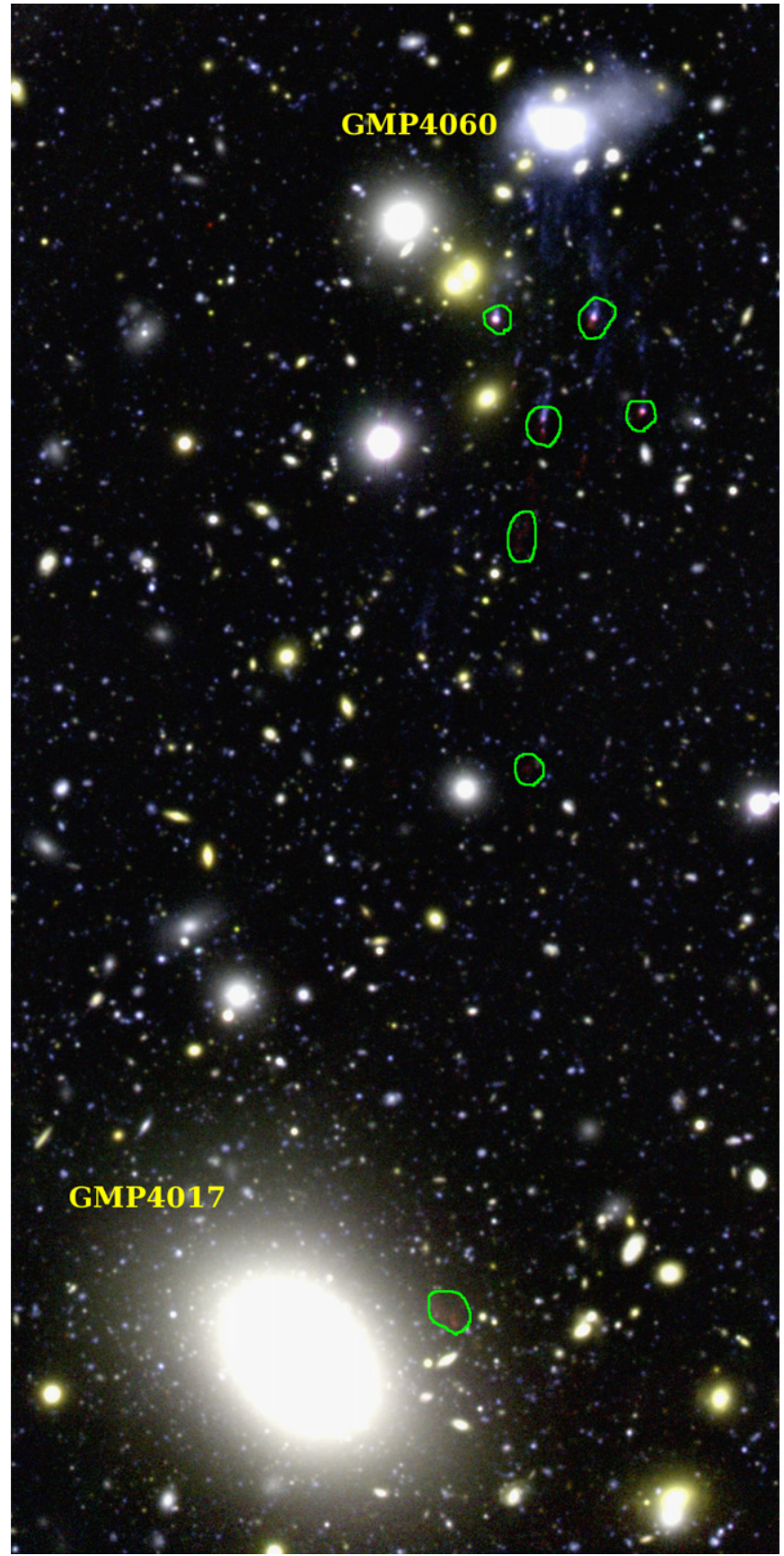

Figure 5. Three-color composite between GMP4060 and GMP4017. The contrast is the same as in Figure $4(\mathrm{~K}$, left) and the stellar streams seen in Figure 4(1) are difficult to recognize. Noticeable $\mathrm{H} \alpha$ clouds are marked by green open shapes.

2000, 2001), with rms per channel of 0.20-0.40 mJy beam ${ }^{-1}$. Although the survey covered the entire field of this study, there were only three parents in which H I gas was detected (GMP2374, GMP2559, and GMP3779). Much deeper H i observations may be required to determine whether GMP4017 or GMP4060 is the true parent of the $\mathrm{H} \alpha$ clouds near GMP4017.

\section{RESULTS}

\subsection{Distribution of the Parents in the Cluster}

Figure 6 shows the positions of the parents, the length and the position angle of the clouds projected onto the sky. The redshift relative to the Coma cluster is also indicated as the

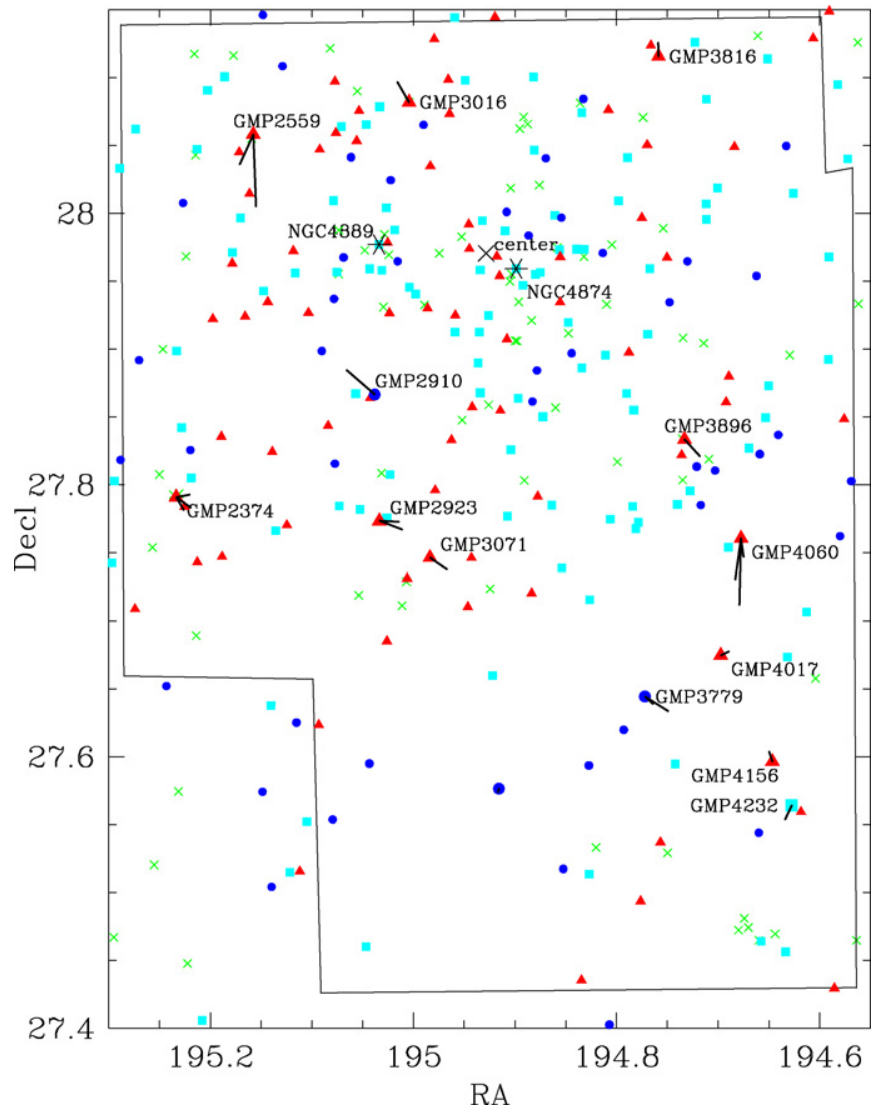

Figure 6. Position angle and extension of $\mathrm{H} \alpha$ clouds in the observed region. The cluster center is shown as a cross and cD galaxies (NGC 4874 and NGC 4889) are shown by the two asterisks. The redshift by SDSS and by Hectospec for the two parents are represented as blue circles $(0.015<z<0.02)$, cyan squares $(0.02 \leqslant z<0.025)$, red triangles $(0.025 \leqslant z<0.035)$, and green crosses (no data), respectively. The parent galaxies are shown as large symbols with solid lines, indicating the direction and distance to the tips of the clouds. The length of the lines is not zoomed and the scale on the margin applies.

color of the symbols. No clouds extend toward the center, although they do not always extend in the anti-center direction either. In addition, there is no apparent sub-clustering of the parents.

Figure 7 shows the redshift of galaxies as a function of the distance from the cluster center. The position of $(\alpha, \delta)=$ $(12: 59: 42.8,+27: 58: 14)$ was adopted as the center of the cluster (White et al. 1993). This coordinate was used in our previous studies (Komiyama et al. 2002; Carter et al. 2002). Figure 7 shows that most of these parent galaxies appear to reside in the blue and red edges of the redshift distribution, suggesting that the parents have large velocities relative to the Coma cluster. We used the Kolmogorov-Smirnov (K-S) test to check whether the distribution of the peculiar velocity with respect to the cluster mean velocity was the same between the parents and the other member galaxies. The sample was restricted in the magnitude range $r<17.8$ and in our observed field to ensure unbiased samples. From the test, the $p$-value for the null hypothesis that the two distributions are the same was calculated as 0.038 . Therefore, the distribution of the parents' redshift is significantly different from that of other member galaxies with over $95 \%$ confidence.

The parents are also located in the region farther than $0.2 \mathrm{Mpc}$ from the cluster center avoiding the cluster core. The difference in the distribution of distance was also examined by the K-S 

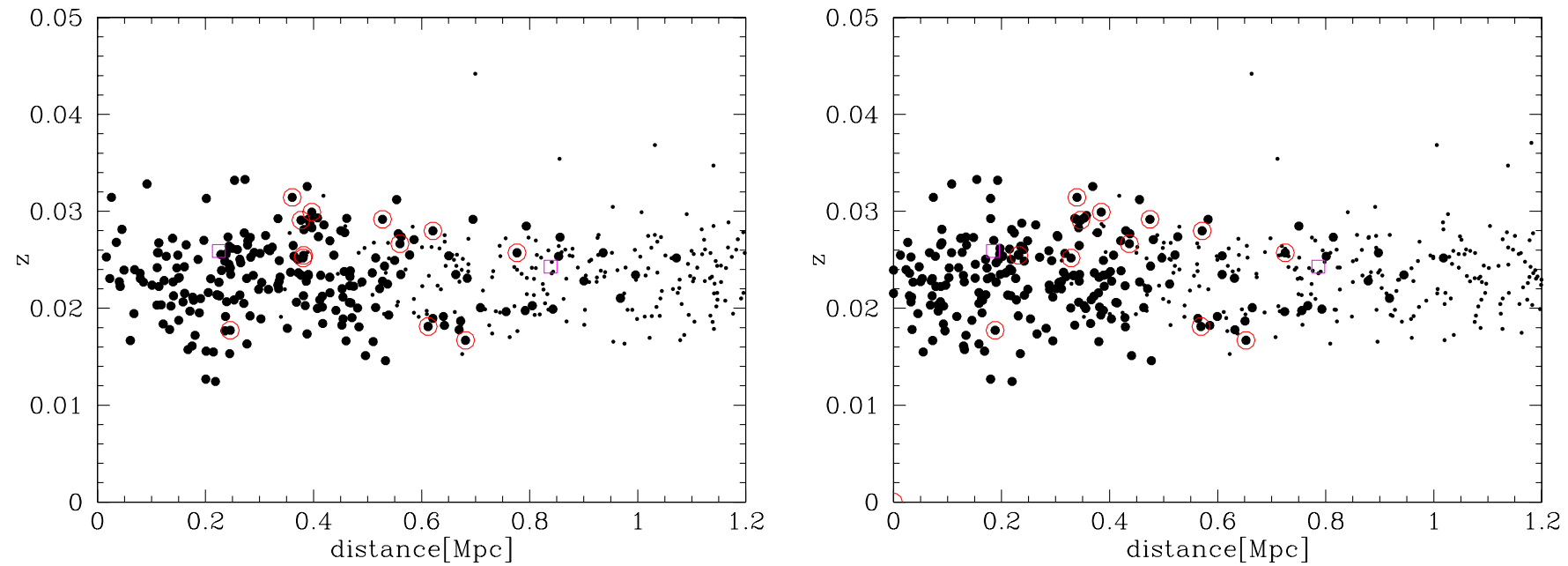

Figure 7. Left: redshift vs. the distance from the cluster center. All galaxies brighter than $r=17.8$ mag and with SDSS spectroscopic data within 42.2 arcmin (which corresponds to $1.2 \mathrm{Mpc}$ at the Coma distance) from the Coma cluster center are plotted as small dots. The galaxies in our observed regions are shown as filled circles. The 12 parent galaxies with SDSS spectra are marked with red open circles, and the two parent galaxies with Hectospec spectra are marked with magenta open squares. Right: same as left, except the distance from the nearest cD is on the abscissa.

(A color version of this figure is available in the online journal.)

test. The distribution of parent galaxies was different from that of other member galaxies ( $p$-value $=0.045$ )

Figure 7 shows that only three parents lie on the blueshifted side while the rest lie on the redshifted side. The 11 redshifted parents could be part of a group falling into the cluster (Poggianti et al. 2004). However, we could not reject the possibility that the parents are falling into the cluster randomly from both sides, because the probability that either the redshifted or blueshifted group has less than 4 of 14 members is $5.7 \%$, given the assumption of random infall.

\subsection{Color of Parents}

As the parent galaxies are bright, the centers of some of the galaxies were saturated in our Subaru images. As the photometric data for these galaxies were not reliable, we took the photometric data from SDSS DR7. Figure 8 shows the color-magnitude diagrams of the galaxies in our observed field. The filled circles represent the SDSS galaxies with $0.015<z<$ 0.035 and $r$-band magnitude $<18.0$.

It is striking that more than half of the blue galaxies are parents of some $\mathrm{H} \alpha$ clouds. The color-magnitude relation (CMR) of passive member galaxies in the Coma cluster was fitted as

$$
g-r=-0.0312 r+1.233 \text {. }
$$

If we define "blue galaxies" as those bluer by $>0.2$ mag than the CMR. The fraction of parents in the blue galaxies is $57 \%$ $(8 / 14)$ in the $r<17.8 \mathrm{mag}\left(M_{r}<-17.25\right)$ SDSS confirmed members.

In the SDSS DR7 photometric catalog, there are nine objects in our observed field which are classified as galaxies, have a "blue" color, are brighter than $r<17.8 \mathrm{mag}$, and have no SDSS redshift. We checked the images of those objects and found that they consisted of three bright stars, one ghost of a bright object, two parts of other galaxies, and three galaxies. Two of the three galaxies, GMP3016 and GMP4232, are members, which is confirmed by Hectospec spectroscopy, and had $\mathrm{H} \alpha$ tails as shown in Figure 4. The remaining one was part of the GMP2863 complex. GMP2863 consists of at least three components, which we call GMP2863a, GMP2863b, and GMP2863c; of these, GMP2863b has a blue color in SDSS. If GMP2863b is a

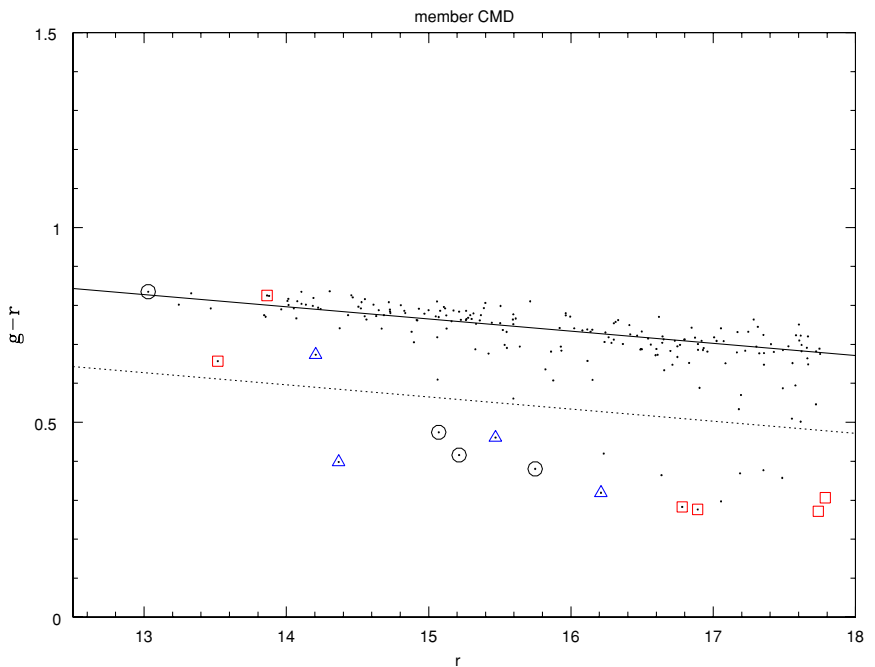

Figure 8. Color-magnitude diagram of member galaxies brighter than $r=$ $17.8 \mathrm{mag}$ in SDSS. The large symbols show the parent galaxies of the $\mathrm{H} \alpha$ clouds. The squares without a dot represent GMP3016 and GMP4232, for which spectroscopic data are unavailable in SDSS. The colors and symbols represent the morphological type of the parent-cloud connection; black open circles (1: connected $\mathrm{H} \alpha$ clouds with disk star formation), blue open triangles (2: connected $\mathrm{H} \alpha$ clouds without disk star formation), and red squares (3: detached $\mathrm{H} \alpha$ clouds). The solid line is the best-fit of the color-magnitude relation (CMR) of passive galaxies. The broken line is the $0.2 \mathrm{mag}$ shifted CMR, which is a demarcation line between blue and red galaxies.

(A color version of this figure is available in the online journal.)

member, the fraction of parents in the blue galaxies is $59 \%$ $(10 / 17)$; if it is not, the fraction is $63 \%(10 / 16)$.

It should be noted that we used an apparent $g-r$ color in this analysis. As we did not correct for internal dust extinction of the parent galaxy in this study, their intrinsic color may be bluer.

\subsection{Morphology of the Cloud-Parent Connection}

The distributions of $\mathrm{H} \alpha$ clouds around the parent (Figures 4(a)-(n)) were varied. We classified them into three types based on their appearance: (1) connected $\mathrm{H} \alpha$ clouds with disk star formations, (2) connected $\mathrm{H} \alpha$ clouds without disk star 
formation, and (3) detached $\mathrm{H} \alpha$ clouds. The results are listed in Table 3.

(1) Connected $\mathbf{H} \alpha$ clouds with disk star formation: GMP2374, GMP3271, and GMP3816 show galaxy-wide star formation and some regions of $\mathrm{H} \alpha$ emission have flown out of the galaxies entirely. GMP3271 and GMP3816 are blue, while GMP2374 is as red as early-type galaxies and lies on the tip of the CMR. Nevertheless, GMP2374 exhibits a very clear spiral pattern with ongoing star formation at the arms. We suppose that GMP2374 could be a progenitor of a passive spiral galaxy (e.g., Goto et al. 2003). GMP2559 has negative H $\alpha$ on the northern side, but strong $\mathrm{H} \alpha$ emission on the southern side. We also classified it as belonging to this type.

(2) Connected $\mathbf{H} \alpha$ clouds without disk star formation: GMP2910, GMP3071, and GMP3779 exhibit core star formation and extended $\mathrm{H} \alpha$ emission connected to the core. The extended clouds have a blobby structure. GMP3896 has a similar appearance to type \#1, but with more extended emission in the disk. Their color and magnitude are similar to those of type \#1.

(3) Detached H $\alpha$ clouds: GMP2923, GMP3016, GMP4060, and GMP4232 have no apparent $\mathrm{H} \alpha$ emission in the parent galaxy, only outside the galaxy. The color of the parent is very blue $(g-r=0.27-0.30)$, and the magnitude is very faint $(r>16.8)$. The four galaxies are in fact the four least luminous galaxies among the parents. GMP3016 and GMP4060 show fireball features: $\mathrm{H} \alpha$ emission following knots of young blue stars (Yoshida et al. 2008).

The clouds of GMP4017 and GMP4156 are also detached. However, the emission is superimposed on the stellar component of the parent galaxy, and the parents are red. The clouds near the two giant galaxies could be different from those near the other four dwarfs. We classified GMP2923, GMP3016, GMP4060, and GMP4232 as \#3 blue dwarfs, and GMP4017 and GMP4156 as \#3 red giants. The central $R-N B$ excess of GMP4156 was partly due to the saturation of our $R$-band image and red color of stellar components, although $\mathrm{H} \alpha$ emission existed in the SDSS spectrum of 3 arcsec aperture. The SDSS spectrum showed a high $[\mathrm{N}$ II $] / \mathrm{H} \alpha$ ratio of $\sim 0.85$, which may indicate a weak active galactic nucleus (AGN).

We noticed that all of the \#3 blue dwarfs showed post-starburst signatures in their SDSS/Hectrospec spectra. As the fiber sizes were $3 \operatorname{arcsec}(1.4 \mathrm{kpc})$ and $1.5 \operatorname{arcsec}(0.7 \mathrm{kpc})$ for SDSS and Hectospec, respectively; their central regions are in a poststarburst phase. Moreover, none of the \#3 blue dwarfs were detected in deep radio $1.4 \mathrm{GHz}$ observations with the Very Large Array (Miller et al. 2009). This suggests that they now have little or no star formation even in their outer regions. Meanwhile, seven of eight \#1 and \#2 parents were detected in radio; these are star-forming galaxies and not radio-loud AGNs.

\section{DISCUSSION}

\subsection{Possible Evolution Sequence of the Parent Galaxies}

The findings presented in Sections 4.1 and 4.2 suggest that the parent galaxies were recently captured by the Coma cluster potential and are now infalling toward the cluster center, while their disk gas is stripped off and seen as $\mathrm{H} \alpha$ clouds.

The classification based on apparent morphology may imply an evolutionary sequence of gas stripping: a star-forming disk galaxy (\#1) evolves into star-forming + dead disk phase (\#2) and then the gas clouds are detached (\#3). Recent numerical simulations of ram-pressure stripping (Kronberger et al. 2008; Kapferer et al. 2009; Tonnesen \& Bryan 2010) also showed
Table 4

Mass Estimation of Parent Galaxies ${ }^{\mathrm{a}}$

\begin{tabular}{cccccccc}
\hline \hline $\begin{array}{c}\text { Name } \\
(1)\end{array}$ & $\begin{array}{c}r-i \\
(2)\end{array}$ & $\begin{array}{c}i \\
(3)\end{array}$ & $\begin{array}{c}\log (M / L)_{i} \\
(4)\end{array}$ & $\begin{array}{c}M_{\text {Bell }} \\
(5)\end{array}$ & $\begin{array}{c}M_{\text {kcorrect }} \\
(6)\end{array}$ & $\begin{array}{c}M_{\text {MPA }} \\
(7)\end{array}$ & $\begin{array}{c}\text { Type } \\
(8)\end{array}$ \\
\hline GMP2374 & 0.43 & 12.6 & 0.34 & 1300 & $630^{\mathrm{b}}$ & 1500 & 1 \\
GMP2559 & 0.32 & 14.8 & 0.21 & 130 & 43 & 100 & 1 \\
GMP2910 & 0.23 & 15.2 & 0.11 & 73 & 12 & 21 & 2 \\
GMP2923 & 0.16 & 16.7 & 0.04 & 16 & 4 & 8 & 3 \\
GMP3016 & 0.18 & 17.6 & 0.06 & 7 & 1 & $\ldots$ & 3 \\
GMP3071 & 0.18 & 16.0 & 0.05 & 31 & 11 & 19 & 2 \\
GMP3271 & 0.17 & 15.6 & 0.05 & 44 & 7 & 9 & 1 \\
GMP3779 & 0.21 & 14.2 & 0.09 & 180 & 33 & 47 & 2 \\
GMP3816 & 0.27 & 14.9 & 0.16 & 110 & 56 & 100 & 1 \\
GMP3896 & 0.39 & 13.8 & 0.29 & 400 & 93 & 350 & 2 \\
GMP4017 & 0.42 & 13.4 & 0.32 & 630 & 300 & 730 & $3(\mathrm{red})$ \\
GMP4060 & 0.17 & 16.6 & 0.04 & 17 & 4 & $\ldots$ & 3 \\
GMP4156 & 0.39 & 13.1 & 0.29 & 770 & 240 & 660 & $3(\mathrm{red})$ \\
GMP4232 & 0.13 & 17.6 & 0.01 & 6 & 1 & $\ldots$ & 3 \\
\hline
\end{tabular}

Notes.

a Column 1: name of the parent, Column 2: $r-i$ color, Column 3: $i$ magnitude, Column 4: $\log _{10}$ of $i$-band stellar mass-to-light ratio, Column 5: estimated stellar mass $\left(10^{8} M_{\odot}\right)$ using Bell et al. (2003) formula, Column 6: estimated stellar mass using kcorrect v4_2 (Blanton \& Roweis 2007), Column 7: MEDIAN stellar mass in MPA-JHU SDSS catalog, and Column 8: type.

b GALEX pipeline divided the galaxy into multiple components. Their flux values were combined for the total UV magnitude.

${ }^{c}$ No FUV detection. We used FUV $=25$ with a large (9.99) error.

the evolution of $\mathrm{H} \alpha$ appearance in $~ 500$ Myr. However, it is puzzling that \#3 phase galaxies consist of less luminous blue galaxies and bright red giants. Moreover, GMP2374, the brightest \#1 galaxy, shows a red color. Although part of the red color is due to possible extinction by internal dust, the spectrum of the whole galaxy in the GOLDMINE database (Gavazzi et al. 2003) indicates that it is dominated by intermediate and old stellar populations as inferred from its large D4000 and small $\mathrm{CaHK}$ ratio. Therefore, it is difficult to assume that this galaxy will evolve into a post-starburst phase with a blue color. The parents may consist of several kinds of galaxies on different evolutionary paths.

As a second parameter of various evolutionary paths, we estimated the masses of the parents. Higher mass galaxies should have larger potential wells and retain some gas for star formation, while lower mass galaxies might simply evolve from type \#1 to \#3 without passing through \#2.

The masses of the parents were estimated by three different methods and are given in Table 4. Bell et al. (2003) give stellar mass-to-light ratios $(M / L)$ as a function of color. They adopted a "diet" Salpeter initial mass function (IMF), which has $30 \%$ lower mass than Salpeter IMF (Salpeter 1955). We adopted

$$
\log _{10}(M / L)_{i \text {-band }}=0.006+1.114(r-i)-0.15
$$

and calculated the stellar mass from the SDSS $r$ and $i$ magnitudes. We neglected $K$-correction for both the $i$ magnitude and $r-i$ colors, which were smaller than $0.1 \mathrm{mag}$. The possible effects of internal dust were also neglected. We also estimated the masses of the parents from the model SED fitting, using kcorrect version 4.1.4 (Blanton \& Roweis 2007) with SDSS and public GALEX magnitudes and redshifts as in Miller et al. (2009). The recommended corrections on the kcorrect Web site ${ }^{11}$ were applied, which included the correction between the AB and SDSS

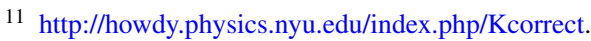


magnitudes, and the minimum uncertainty of the SDSS magnitude. The kcorrect used Chabrier (2003) IMF and Padova 1994 isochrones (Bruzual \& Charlot 2003, and references therein). Yet another mass estimation was available in the MPA-JHU SDSS catalog. ${ }^{12}$ The DR7_v5.2 version included 11 parents of this study. We adopted the "MEDIAN" column as the estimated mass. The IMF by Kroupa (2001) was adopted for the catalog (Kauffmann et al. 2003).

Although there were variations among the mass estimations of the three different methods, we can see that the type \#3 dwarfs had less than $2 \times 10^{9} M_{\odot}$. On the other hand, the classification between \#1 and \#2 showed no clear correlation with mass. In addition, the lowest mass \#3 parents $\left(<2 \times 10^{9} M_{\odot}\right)$ stopped star formation quickly, while most of the \#1 and \#2 parents, the masses of which were $10^{9}-10^{11} M_{\odot}$, continued star formation. The correlation of the detachment of the clouds, the activity of star formation, and the mass implied that the quick quench of the star formation in the \#3 blue dwarfs was due to shallow potentials and/or small amounts of gas. This result regarding the dependence of the star formation quench on the mass was similar to those in previous studies (Kauffmann et al. 2004; Haines et al. 2007; Wolf et al. 2009; Mahajan et al. 2010), although this is the first time that galaxies of low mass $\left(<10^{9} M_{\odot}\right)$ have been investigated in this way.

\subsection{Comparison with Galaxies with UV Asymmetries}

Recently Smith et al. (2010) investigated UV and $\mathrm{H} \alpha$ images of the Coma cluster, and listed the galaxies with gaseous stripping events (GSEs), which were detected as UV asymmetries and had colors with $N U V-i<4$. Their observed field overlapped with our field, and there were six galaxies in common (GMP2559, GMP2910, GMP3016, GMP3816, GMP4060, and GMP4232). It is striking that the UV asymmetric galaxies in our fields are only the six. This suggests that GSE galaxies have extended $\mathrm{H} \alpha$ clouds with high probability.

The other eight parents were not listed as GSE galaxies, although all of the parents in this study satisfied the selection criteria of Smith et al. (2010) $(i<18$, and $0.01<z<0.043)$. As the NUV-optical color is a function of the star formation strength and the age since the last star formation (e.g., Kaviraj et al. 2007), one possibility is that the non-GSE parents are older. This can explain the red \#3 galaxies, as age quickly makes the $g-r$ color redder. The other five star-forming galaxies and a post-starburst galaxy have young stars, and the age cannot explain the non-detection of UV asymmetry. It is possible that the UV asymmetric feature is faint because the star formation is/was weaker than other cases. It is also possible that the UV emitting stars have a symmetric distribution, while the $\mathrm{H} \alpha$ is asymmetric. The last case suggests that the distributions of the young stars and gas differ. The sign of the different distribution was shown in our previous studies (Yagi et al. 2007; Yoshida et al. 2008). This also suggests that the source of ionization is not always the UV photons from young stars. A detailed discussion of the source of ionization will be presented in our next paper (M. Yoshida et al. 2010, in preparation).

If we assume that all galaxies from which gas was removed experienced an $\mathrm{H} \alpha$ parent phase, we can speculate on the evolution of the parents. With regard to infalling galaxies, Graham et al. (2003) investigated two disk dwarf galaxies in the Coma cluster, which still show spiral arm patterns. The galaxies GMP3292 and GMP3629 are thought to be transforming from

\footnotetext{
12 http://www.mpa-garching.mpg.de/SDSS/DR7/
}

spiral to passive galaxies. Graham et al. (2003) also noted that these galaxies have large peculiar velocities relative to the Coma center. However, these two galaxies are much redder than parents in this study $(r=16.5, g-r=0.68$ for GMP3292 and $r=18.3, g-r=0.64$ for GMP3629), but are of comparable mass $\left(4.1 \times 10^{9}\right.$ and $\left.8 \times 10^{8} M_{\odot}\right)$, with values estimated in the same way as for the parents. They might be recently evolved shapes from the parents, as they still retain the arm pattern.

\section{SUMMARY}

From the Suprime-Cam observation of the center of the Coma cluster, we found ionized $\mathrm{H} \alpha$ clouds near 14 galaxies (parents). All the parents were confirmed to be Coma member galaxies by SDSS and Hectospec spectroscopy. The parent galaxies were distributed in the region farther than $0.2 \mathrm{Mpc}$ from the cluster center, and tended to reside on the blue and red edges of the redshift distribution, avoiding the mean cluster redshift. The majority of the parent galaxies were blue, and $57 \%$ of the blue galaxies brighter than $r=17.8$ contained $\mathrm{H} \alpha$ clouds.

We classified the connection between the parent and the clouds into three types: connected clouds with disk star formation, connected clouds without disk star formation, and detached clouds. The parents of detached clouds consisted of two groups; blue dwarfs and red giants. The blue dwarfs were the four least luminous galaxies among the 14 parents, and showed no sign of current star formation but appeared to be post-starburst. The parents with connected clouds showed no significant differences regardless of whether they had disk star formation or not.

A comparison with galaxies with UV asymmetries showed that all such galaxies are accompanied with $\mathrm{H} \alpha$ clouds. This suggests that a galaxy undergoing a GSE has extended $\mathrm{H} \alpha$ emitting clouds with high probability.

We thank the anonymous referee for suggestions and comments that improved this paper. We thank Dr. Russel J. Smith for information on their GSE galaxies and the suggestive comments, Dr. H. Yamanoi for discussion on the photometric calibration of the Suprime-Cam data, and Dr. M. Sun for informing us about their latest results. This work was based on data collected using the Subaru telescope, which is operated by NAOJ. We are grateful to the Subaru staff for their help. This work was supported by KAKENHI 21540247. S.J. acknowledges support from the National Aeronautics and Space Administration (NASA) LTSA grant NAG5-13063, NSF grant AST-0607748, and HST grant G0-10861 from STScI, which is operated by AURA, Inc., for NASA, under NAS5-26555. This work made use of SAOImage DS9 (Joye \& Mandel 2003), the NASA's Astrophysics Data System, ${ }^{13}$ the NASA/IPAC Extragalactic Database (NED), ${ }^{14}$ the SDSS skyserver, ${ }^{15}$ and the data analysis system at the Astronomy Data Center of NAOJ. ${ }^{16}$

\section{APPENDIX}

\section{SUPRIME-CAM FLUX CALIBRATION USING SDSS}

We took special care with the flux calibration. The $B$-band of the Suprime-Cam has different transmission from the standard Johnson- $B$ because of the low throughput of CCDs at shorter wavelengths. In addition, the $R$-band of the Suprime-Cam has

\footnotetext{
13 http://ads.nao.ac.jp/

14 http://nedwww.ipac.caltech.edu/

15 http://cas.sdss.org/

16 http://www.adc.nao.ac.jp/
} 

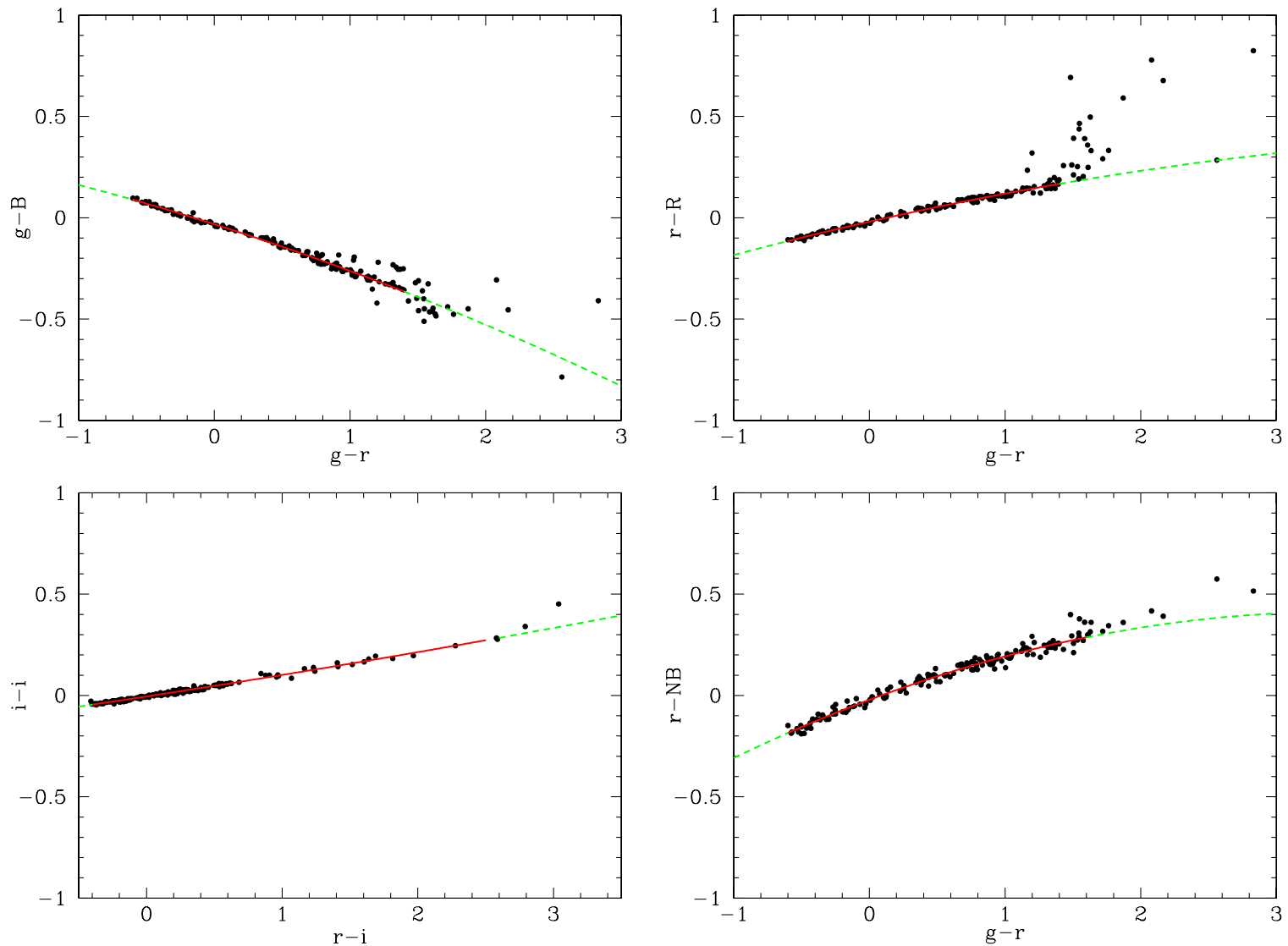

Figure A1. SDSS color vs. SDSS-Suprime color of BPGS stars for old Suprime-Cam CCDs. The line represents the regression curve of the relation, where the range used in the calibration is shown as a red solid line and the other part is shown as a green broken line.

(A color version of this figure is available in the online journal.)

Table A1

The Coefficients of Best-fit Color Conversion Polynomials

\begin{tabular}{lccccc}
\hline \hline SDSS-Suprime & SDSS Color & Range & $c_{0}$ & $c_{1}$ & $c_{2}$ \\
\hline$g-B$ (new) & $g-r$ & $-0.6<g-r<1.4$ & -0.0232335 & -0.174584 & -0.0138875 \\
$g-B$ (old) & $g-r$ & $-0.6<g-r<1.4$ & -0.0317803 & -0.212379 & -0.0178932 \\
\hline$r-R$ (new) & $g-r$ & $-0.6<g-r<1.4$ & -0.0188297 & 0.149276 & -0.0128141 \\
$r-R$ (old) & $g-r$ & $-0.6<g-r<1.4$ & -0.0191869 & 0.151382 & -0.012914 \\
\hline$i-i$ (new) & $r-i$ & $-0.4<r-i<2.5$ & -0.00488511 & 0.101026 & 0.00341974 \\
$i-i$ (old) & $r-i$ & $-0.4<r-i<2.5$ & -0.00472411 & 0.102785 & 0.00326376 \\
\hline$r-N B$ (new) & $g-r$ & $-0.6<g-r<1.6$ & -0.0214062 & 0.250467 & -0.0361111 \\
$r-N B$ (old) & $g-r$ & $-0.6<g-r<1.6$ & -0.021407 & 0.250514 & -0.0361369 \\
\hline
\end{tabular}

different transmission from Kron-Cousins- $R$ as it has a much shallower range. ${ }^{17}$

We adopted the SDSS DR7 catalogs (Abazajian et al. 2009), which cover the whole of our observation area. As many stars in the observed field had photometric data in SDSS DR7, we calibrated the zero point of our data to achieve the best match. We retrieved type $=6$ (stellar objects) of $r<21$ objects for the calibration. The colors of typical stars were calculated using the Bruzual-Persson-Gunn-Stryker Atlas. ${ }^{18}$ Using the filter transmission of SDSS DR7 and that of the Suprime-Cam filters, we plotted $g-B$ (Suprime) versus $g-r, r-R$ (Suprime) versus $g-r$, and $i-i$ (Suprime) versus $r-i$. Then, we fitted the loci

\footnotetext{
$\overline{17}$ http://www.naoj.org/Observing/Instruments/SCam/sensitivity.html

18 ftp://ftp.stsci.edu/cdbs/grid/bpgs/
}

Table A2

The Maximum Color Difference of BPGS Stars in Old and New CCDs

\begin{tabular}{lc}
\hline \hline SDSS-Suprime & Difference \\
\hline$g-B$ & 0.12 \\
$r-R$ & 0.01 \\
$i-i$ & 0.02 \\
$r-N B$ & 0.00 \\
\hline
\end{tabular}

of stars with a second-order polynomial as

$$
\text { SDSS }- \text { Suprime }=\mathrm{c}_{0}+\mathrm{c}_{1}(\text { color })+\mathrm{c}_{2}(\text { color })^{2} .
$$

The coefficients are shown in Table A1, and the fit is also shown in Figures A1 and A2. 

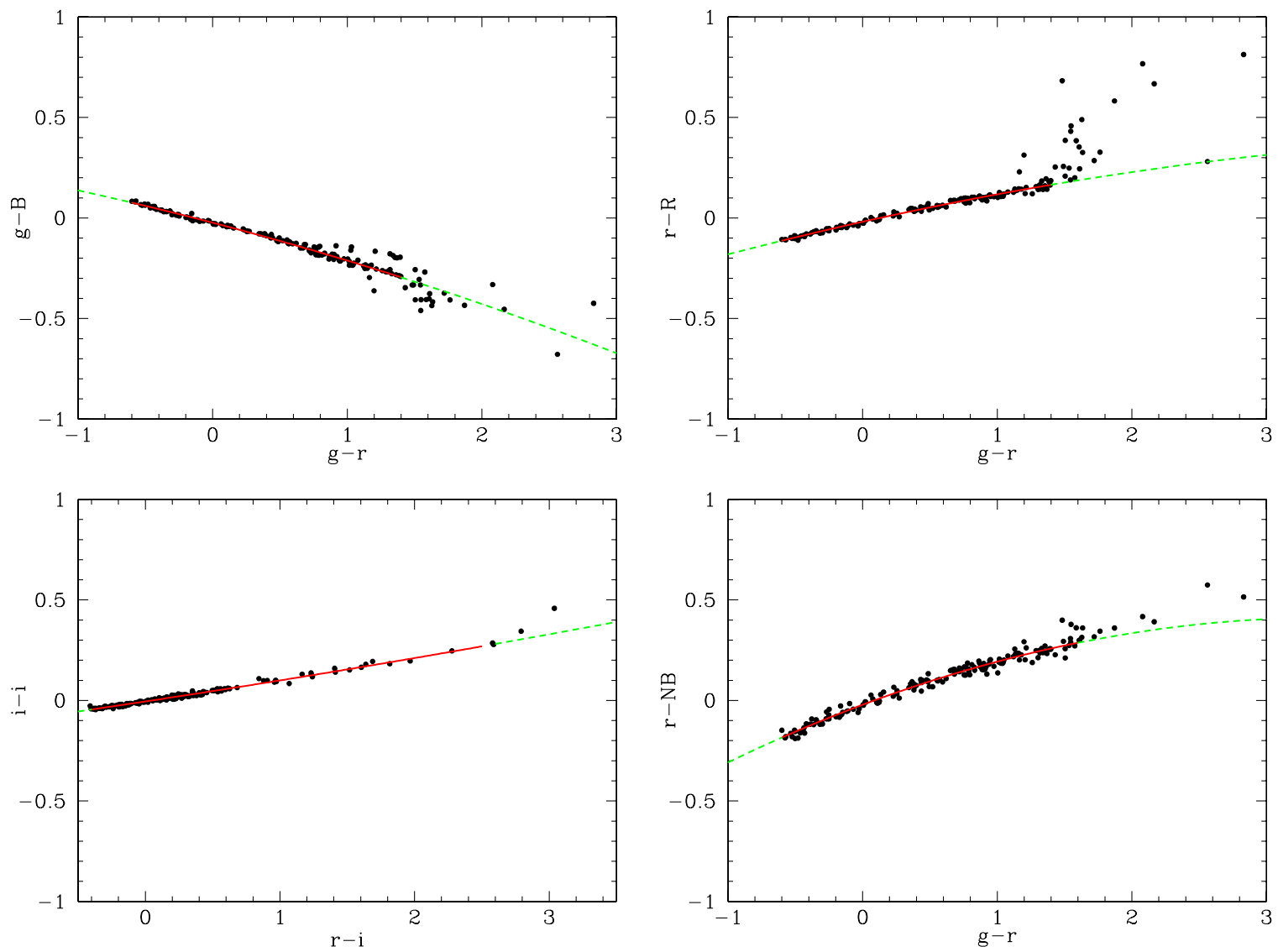

Figure A2. Same as Figure A1 for new Suprime-Cam CCDs.

(A color version of this figure is available in the online journal.)

Replacement of the Suprime-Cam CCDs in 2008 July (Kamata et al. 2008) affected the color terms. The systematic differences between the old and new CCDs are negligible $(<0.01 \mathrm{mag}$ ) for the $R$ and $i$ bands, but $\sim 0.05 \mathrm{mag}$ for the $B$ band (Table A2). This was because of the change in quantum efficiency at $\lambda<4500 \AA$. Therefore, we made two $B$-band images; one from old CCD data and the other from new CCD data. As the latter covers a part of the field, the three color images in this paper were created with the old CCD $B$-band data.

The cross-identification of stars in each FITS data set was performed using celestial coordinates. We used wcstools (Mink 2002) and the USNO-B1.0 catalog (Monet et al. 2003) to calibrate the world coordinate system. The flux measurement was performed with SExtractor (Bertin \& Arnouts 1996). Removing saturated or blended objects, we estimated the shift between the observed data and the model locus using the mode of the difference.

The AB magnitude of the $N B$-band was also calibrated with SDSS in the same manner. We assumed that most of the stars did not have strong features around $6710 \pm 100 \AA$, and hence that the $r-N B$ (Suprime) versus $g-r$ plot would work. We also used spectroscopic data of stars in our observed field to calculate $r-N B$ (Suprime) color. The values were consistent with the model locus of BPGS stars.

This method is applicable to any Suprime-Cam data where SDSS has a photometric catalog. However, this method has the drawback that Galactic extinction may add systematic error, because the model locus is free from the extinction. In the Coma region, however, the Galactic extinction for extragalactic objects is $A_{B}=0.035$, from the model of Schlegel et al. (1998) in NED. The extinction for Galactic stars must be much less than this value, and therefore we neglected the effect in this study.

\section{REFERENCES}

Abazajian, K. N., et al. 2009, ApJS, 182, 543

Bell, E. F., McIntosh, D. H., Kats, N., \& Weinberg, M. D. 2003, ApJS, 149, 289 Bertin, E., \& Arnouts, S. 1996, A\&AS, 317, 393

Blanton, M. R., \& Roweis, S. 2007, AJ, 133, 734

Boselli, A., \& Gavazzi, G. 2006, PASP, 118, 517

Bravo-Alfaro, H., Caretta, C. A., Lobo, C., Durret, F., \& Scott, T. 2009, A\&A, 495, 379

Bravo-Alfaro, H., Cayatte, V., van Gorkom, J. H., \& Balkowski, C. 2000, AJ, 119,580

Bravo-Alfaro, H., Cayatte, V., van Gorkom, J. H., \& Balkowski, C. 2001, A\&A, 379,347

Bruzual, G., \& Charlot, S. 2003, MNRAS, 344, 1000

Byrd, G., \& Valtonen, M. 1990, ApJ, 350, 89

Carter, D., et al. 2002, ApJ, 567, 772

Chabrier, G. 2003, PASP, 115, 763

Cortese, L., et al. 2007, MNRAS, 376, 157

Cowie, L. L., \& Songaila, A. 1977, Nature, 266, 501

Gavazzi, G. 1987, ApJ, 320, 96

Gavazzi, G., Boselli, A., Donati, A, Franzetti, P., \& Scodeggio, M. 2003, A\&A, 400,451

Gavazzi, G., Boselli, A., Mayer, L., Iglesias-Paramo, J., Vílchez, J. M., \& Carrasco, L. 2001, ApJ, 563, L23

Giovanelli, R., \& Haynes, M. P. 1985, ApJ, 292, 404

Godwin, J. G., Metcalfe, N., \& Peach, J. V. 1983, MNRAS, 202, 113

Goto, T., et al. 2003, PASJ, 55, 757

Graham, A. W., Jerjen, H., \& Guzmán, R. 2003, AJ, 126, 1787 
Gunn, J. E., \& Gott, J. R. I. 1972, ApJ, 176,

Haines, C. P., Gargiulo, A., La Barbera, F., Mercurio, A., Merluzzi, P., \& Busarello, G. 2007, MNRAS, 381, 7

Joye, W.A, \& Mandel, E. 2003, in ASP Conf. Ser. 295, ADASS XII, ed. H. E. Payne, R. I. Jedrzejewski, \& R. N. Hook (San Francisco, CA: ASP), 489

Kamata, Y., et al. 2008, Proc. SPIE, 7021, 52

Kapferer, W., Sluka, C., Schindler, S., Ferrari, C., \& Ziegler, B. 2009, A\&A, 499,87

Kashikawa, N., et al. 2002, PASJ, 54, 819

Kauffmann, G., White, S. D. M., Heckman, T. M., Ménard, B., Brinchmann, J., Charlot, S., Tremonti, C., \& Brinkmann, J. 2004, MNRAS, 353, 713

Kauffmann, G., et al. 2003, MNRAS, 341, 33

Kavelaars, J. J., Harris, W. E., Hanes, D. A., Hesser, J. E., \& Pritchet, C. J. 2000, ApJ, 533, 125

Kaviraj, S., Kirkby, L. A., Silk, J., \& Sarzi, M. 2007, MNRAS, 382, 960

Kenney, J. D. P., Tal, T., Crowl, H. H., Feldmeier, J., \& Jacoby, G. H. 2008, ApJ, 687, L69

Komiyama, Y., et al. 2002, ApJS, 138, 265

Kronberger, T., Kapferer, W., Ferrari, C., Unterguggenberger, S., \& Schindler, S. 2008, A\&A, 481, 337

Kroupa, P. 2001, MNRAS, 322, 321

Larson, D., et al. 2010, arXiv:1001.4635

Mahajan, M., Haines, C. P., \& Raychaudhury, S. 2010, MNRAS, 404, 1745

Miller, N. A., Hornschemeier, A. E., Mobasher, B., Bridges, T. J., Hudson, M. J., Marzke, R. O., \& Smith, R. J. 2009, AJ, 137, 4450

Mink, D. J. 2002, in ASP Conf. Proc. 281, ADASS XI, ed. D. A. Bohlender, D. Durand, \& T. H. Handley (San Francisco, CA: ASP), 169

Miyazaki, S., et al. 2002, PASJ, 54, 833

Monet, D. G., et al. 2003, AJ, 125, 984
Moore, B., Katz, N., Lake, G., Dressler, A., \& Oemler, A. 1996, Nature, 379, 613

Nulsen, P. E. J. 1982, MNRAS, 198, 1007

Okabe, N., Okura, Y., \& Futamase, T. 2010, ApJ, 713, 201

Oosterloo, T., \& van Gorkom, J. 2005, A\&A, 437, L19

Poggianti, B. M., Bridges, T. J., Komiyama, Y., Yagi, M., Carter, D., Mobasher, B., Okamura, S., \& Kashikawa, N. 2004, ApJ, 601, 197

Salpeter, E. E. 1955, ApJ, 121, 161

Schlegel, D. J., Finkbeiner, D. P., \& Davis, M. 1998, ApJ, 500, 525

Smith, R. J., et al. 2010, MNRAS, 408, 1417

Solanes, J. M., Manrique, A., García-Gómez, C., González-Casado, G., Giovanelli, R., \& Haynes, M. P. 2001, ApJ, 548, 97

Sun, M., Donahue, M., \& Voit, M. 2007, ApJ, 671, 170

Sun, M., Donahue, M., Roediger, E., Nulsen, P. E. J., Voit, G. M., Sarazin, C., Forman, W., \& Jones, C. 2010, ApJ, 708, 964

Tonnesen, S, \& Bryan, G. L. 2010, ApJ, 709, 1203

Toomre, A., \& Toomre, J. 1972, ApJ, 178, 623

van der Wel, A., Bell, E. F., Holden, B. P., Skibba, R. A., \& Rix, H.-W. 2010, ApJ, 714,1779

White, S. D. M., Briel, U. G., \& Henry, J. P. 1993, MNRAS, 261, L8

Wolf, C., et al. 2009, MNRAS, 393, 1302

Yagi, M., Kashikawa, N., Sekiguchi, M., Doi, M., Yasuda, N., Shimasaku, K., \& Okamura, S. 2002, AJ, 123, 66

Yagi, M., Komiyama, Y., Yoshida, M., Furusawa, H., Kashikawa, N., Koyama, Y., \& Okamura, S. 2007, ApJ, 660, 1209

Yagi, M. 2010, in ASP Conf. Ser. 434, ADASS XIX, ed. Y. Mizumoto, K.-I. Morita, \& M. Ohishi (San Francisco, CA: ASP), 465

Yoshida, M., et al. 2002, ApJ, 567, 118

Yoshida, M., et al. 2008, ApJ, 688, 918 